\title{
Efficient Inference of Sensitivity to Electrical Stimulation from Intrinsic Electrical Properties of Primate Retinal Ganglion Cells
}

\author{
Sasi S. Madugula* 3,4,7,10, Lauren E. Grosberg ${ }^{1,7,9}$, Nishal P. Shah ${ }^{1,2,7}$, Alexandra Kling ${ }^{1,7}$, Alex R. Gogliettino ${ }^{3,7}$, \\ Paweł Hottowy ${ }^{8}$, Alexander Sher ${ }^{5}$, Alan M. Litke ${ }^{5}$, and E.J. Chichilnisky ${ }^{1,6,7}$ \\ 1. Department of Neurosurgery, Stanford University, Stanford, CA 94305, USA \\ 2. Department of Electrical Engineering, Stanford University, Stanford, CA 94305, USA \\ 3. Neurosciences PhD Program, Stanford University, Stanford, CA 94305, USA \\ 4. School of Medicine, Stanford University, Stanford, CA 94305, USA \\ 5. Santa Cruz Institute for Particle Physics, University of California, Santa Cruz, CA 95064, USA \\ 6. Department of Ophthalmology, Stanford University, Stanford, CA 94305, USA \\ 7. Hansen Experimental Physics Laboratory, Stanford University, Stanford, CA 94305, USA \\ 8. Faculty of Physics and Applied Computer Science, AGH University of Science and Technology, Krakow, Poland \\ 9. Facebook Reality Labs, Facebook, Mountain View, CA 94040, USA \\ 10. Lead Contact \\ ${ }^{*}$ Correspondence: sasidhar@stanford.edu
}

\section{Summary}

High-fidelity sensory neural implants must be calibrated to precisely activate specific cells via extracellular stimulation. However, collecting and analyzing the required electrical stimulation data may be difficult in the clinic. A potential solution is to infer stimulation sensitivity from intrinsic electrical properties. Here, this inference was tested using large-scale high-density stimulation and recording from macaque retinal ganglion cells ex vivo. Electrodes recording larger spikes exhibited lower activation thresholds, with distinct trends for somas and axons, and consistent trends across cells and retinas. Thresholds for somatic electrodes increased with distance from the axon initial segment. Responses were inversely related to thresholds, and exhibited a steeper dependence on injected current for axonal than somatic electrodes. Dendritic electrodes were largely ineffective for eliciting spikes. Biophysical simulations qualitatively reproduced these findings. Inference of stimulation sensitivity was employed in simulated visual reconstruction, revealing that the approach could improve the function of future high-fidelity retinal implants. 


\section{Introduction}

Sensory neural interfaces of the future, such as visual, auditory and somatosensory implants, may operate at cellular resolution, permitting the selective activation of individual cells and cell types to produce high-fidelity artificial sensation. While recording and stimulation hardware continues to advance toward this goal, calibration remains a significant obstacle. Specifically, for any given collection of electrodes and neurons, an implant that recreates the neural code at cellular resolution must rely on knowledge of which electrodes activate which cells, and to what degree. Obtaining this information, in turn, requires measurements of responses to electrical stimulation in large collections of neurons that are in contact with a large-scale neural implant. This calibration raises many problems in the clinic: it is time-consuming, requires complex analysis to distinguish electrical responses from electrical artifacts, may produce uncomfortable and startling artificial sensations, and may need to be performed repeatedly to accommodate tissue rearrangement or device movement. Thus, any intrinsic biophysical properties of neurons that could be readily measured to reveal features of their electrical sensitivity could significantly speed and enhance the calibration of neural interfaces, and therefore could have a large impact on translational neuroengineering.

One potential solution to the calibration problem would be to harness information from recorded spontaneous electrical activity in order to infer how cells will respond to electrical stimulation. Spontaneous activity can be recorded rapidly in parallel on many electrodes and is not subject to electrical artifacts, and therefore can be analyzed relatively easily. An example of this inference comes from the observation that electrodes closer to a particular cell tend to record larger spikes and also typically require less stimulation current to elicit activity. Thus, spike amplitudes recorded during spontaneous activity could potentially be used to infer electrical stimulation thresholds for every electrode and cell. The possibility of this kind of inference has been suggested in theoretical and modeling studies, and experimental work in tissue culture (Boinagrov et al., 2010; Esler et al., 2018a; Fohlmeister et al., 1990; Loizos et al., 2014; Radivojevic et al., 2016; Tsai et al., 2012). However, these approaches have not been tested with real neurons functioning within complete neural circuits, and the modeling work relies on simplified assumptions about the electrical properties of neural tissue and on detailed knowledge of neuronal geometry, which generally cannot be determined in a living patient. Thus, it remains unclear whether and how the electrical sensitivity of neurons in intact tissue can be reliably inferred from their recorded spontaneous activity.

In this paper we propose an approach for electrical sensitivity inference in the context of a future high-resolution retinal implant to treat incurable blindness. A sufficiently precise implant could, in principle, reproduce the neural code of the retina for high-quality artificial vision, but calibrating such a device is a daunting task. We leverage high-density multi-electrode recording and stimulation from hundreds of retinal ganglion cells (RGCs) in the primate retina as a laboratory prototype for such a device, to probe the relationship between recorded spikes and sensitivity to electrical stimulation. The sensitivity of RGCs to extracellular stimulation at various locations depended, in a surprisingly consistent manner across hundreds of electrodes and dozens of retinal preparations, on recorded features of spiking activity across space. This dependence exhibited systematically different properties based on the proximity of each electrode to the soma, axon, and dendrites of the cell, the characteristics of which were in accordance with predictions from biophysical models. These trends were useful in using recorded intrinsic spiking activity to predict the responses of individual RGCs to electrical stimulation. Exploiting these relationships to infer optimal electrical stimulation sequences proved to be effective for enhancing high-fidelity image reconstruction, and thus could be useful for a future high-fidelity retinal implant. 


\section{Results}

The efficacy of closed-loop epiretinal implants, and of electrical neural implants in other regions of the nervous system, could be markedly improved by the ability to infer the responsivity of nearby neurons to extracellular stimulation using only features of recorded spontaneous spikes, which are much faster to obtain and easier to analyze. To perform this inference would require establishing a consistent relationship between recording and stimulation, by comparing spatial signatures of cells obtained by electrical recording alone with their responses to extracellular electrical stimulation, at distinct spatial locations. Below, we explore this relationship for macaque retinal ganglion cells (RGCs).

An array of 512 electrodes (10 $\mu \mathrm{m}$ diameter, $60 \mu \mathrm{m}$ pitch [18]) was used to electrically record and stimulate RGCs in ex vivo isolated macaque retina preparations. Responses of recorded RGCs to white noise visual stimulation were used to identify ON and OFF parasol cells, two of the numerically dominant RGC types in the macaque retina (Dacey, 2004; Field and Chichilnisky, 2007). These cells were then probed with electrical stimulation, eliciting individual, precisely-timed, directly-evoked spikes (Jepson et al., 2013; Sekirnjak et al., 2008). To avoid activation of RGCs off the array, stimulating current amplitudes at each electrode were limited to values lower than the threshold for stimulation of axon bundles (Grosberg et al., 2017; Tandon et al., 2021). Spikes evoked by electrical stimulation were identified separately from the electrical stimulation artifact using an automated approach (Mena et al., 2017). This procedure enabled the collection of paired recording and stimulation data on multiple electrodes for 172 RGCs of the ON and OFF parasol types.

To obtain a spatial signature of spontaneous spikes produced by each recorded cell, the electrical image (El) was computed. The $\mathrm{El}$ is the average spatiotemporal pattern of activity associated with a spike in a single cell (Fig. 1A). The El can be obtained from recordings of spontaneous or visually-evoked activity (see Methods). For a given $\mathrm{El}$, the characteristic shape of the spike waveform at each recording electrode reflects the underlying cellular compartment that contributes most to the signal at that electrode: dendrite, soma, or axon (Litke et al., 2004). An automated method was developed to distinguish these compartments, based on the amplitudes of one or more positive and negative peaks in the recorded spike waveforms (see Methods). The three cellular compartments were spatially distributed in the plane of the retina as expected, based on the known geometry of RGCs (see Methods; Fig. 1), with the identified axons extending toward the optic disc. Additionally, for each El, the putative location of the axon initial segment (AIS), a region on the cell considered to be the most sensitive to electrical stimulation (Boiko et al., 2003; Esler et al., 2018b; Fried et al., 2009; Werginz et al., 2020), was assumed to be $13 \mu \mathrm{m}$ from the soma center in the direction of the nearest axonal electrode, based on previous empirical measurements in the same experimental conditions (Sekirnjak et al., 2008).

To determine the extracellular activation characteristics for each cell at various spatial locations, responses evoked by electrical stimulation at each electrode were characterized to construct an electrical receptive field (ERF). First, responses to current pulses over a range of amplitudes were recorded, and spike waveforms in these recordings were identified and separated from electrical artifacts. These waveforms were then matched to the waveforms of light-evoked spikes (from the El, see above) in order to identify which cell(s) produced a spike in response to the electrical stimulus (see Methods). For each electrode, the relationship between current amplitude and spiking probability was summarized by a sigmoidal activation curve (Fig. 1, bottom row), and the current amplitude that evoked spikes in $50 \%$ of trials was defined as the activation threshold. Finally, the ERF 
for each cell was defined as a spatial map of sensitivity to electrical stimulation, given by the inverse of the activation threshold at each electrode location (Fig. 1).

\section{Features of electrical images correspond to the electrical receptive fields of ON and OFF parasol cells}

Features of the El, a relatively simple measurement, provided substantial information about the ERF (Fig. 1), which summarizes electrical excitability over space but is far more difficult to obtain. Several observations about data from a single cell reveal trends that are discussed in detail subsequently. First, only electrodes that recorded large spike waveforms from a given cell (Fig. 1, hollow circles) were able to evoke spiking in that cell (Fig. 1, filled circles) within the amplitude range tested (5-200 pC injected charge), although many did not activate the cell at current levels below bundle threshold (Fig. 1, light-blue hollow circles; see Methods). Second, for the electrodes that did evoke a spike below bundle threshold (Fig. 1; see Methods), the El amplitude at each electrode was related to the strength of the ERF: electrodes recording higher EI amplitudes (Fig. 1, circle size) also excited the cell more effectively--i.e. with lower thresholds (Fig. 1, circle color). The similarity between Els and ERFs presumably reflects the fact that both spike amplitude and sensitivity to electrical stimulation are inversely related to the distance between the electrode and various compartments of the cell (Rattay, 1987; Rattay et al., 2012; Sekirnjak et al., 2008). Third, somatic electrodes closer to the axon (Fig. 1B, electrode at circle 2) required less current to activate RGCs than somatic electrodes further from the axon (Fig. 1B, electrode at circle 1), even if the electrodes recorded spikes of similar amplitude (Fig. 1B, radii of circles $1 \& 2$ ). Fourth, electrical stimulation using dendritic electrodes typically did not elicit spiking within the tested stimulation amplitude range (Fig. 1A, top \& bottom right). Finally, axonal electrodes exhibited steeper increases of firing probability in response to increasing stimulation amplitude (Fig. 1 bottom, red vs. blue sigmoids, slopes), and recorded spike waveforms with smaller amplitudes (Fig. 1 top, red vs. blue waveforms, negative peak amplitudes), despite exhibiting activation thresholds in a range similar to those of somatic electrodes $(0.2-2 \mu \mathrm{A})$. The latter suggested that the relationship between Els and activation curves differs for somatic and axonal electrodes. In the following, these trends in the relationship between the El and ERF are explored across many cells and retinas.
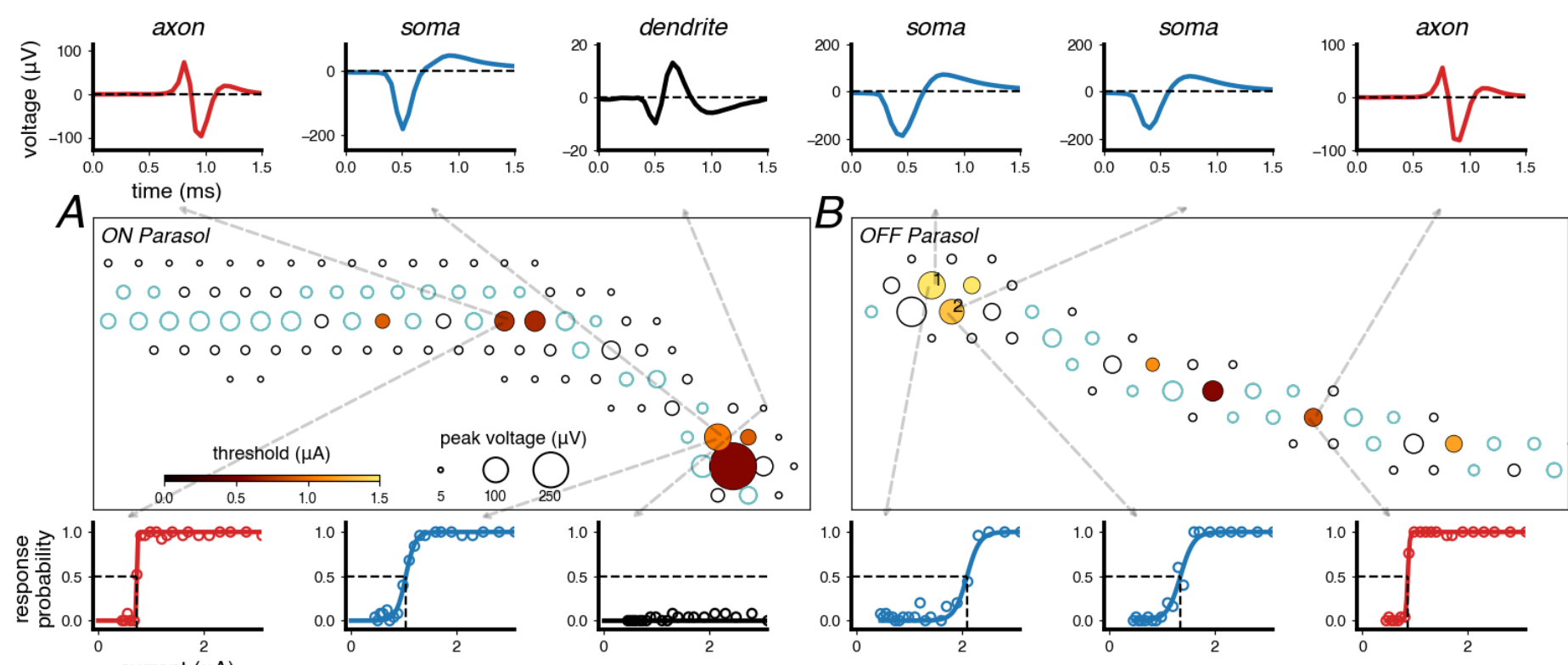

Figure 1. Recording and stimulation in single cells. A) ON Parasol Electrical Image (EI) superimposed on the Electrical Receptive Field $(E R F)$; circle area is proportional to recorded signal strength, and electrode color corresponds to stimulation threshold. Electrodes that recorded spike amplitudes less than the electrical noise threshold $(30 \mu \mathrm{V})$ are not plotted, and those that didn't elicit spikes are black 
and hollow. Electrodes where the signal could not be analyzed due to axon bundle activity are light blue and hollow. Flanking the EI plots are examples of recorded dendritic (black), somatic (blue), and axonal (red) spike waveforms (top; see Methods), and examples of sigmoidal electrical activation curves characterizing measured spiking responses (circles) to current stimulation at each cellular compartment (bottom). B) El superimposed over the ERF for an OFF Parasol cell from the same retina, with example waveforms recorded from the soma at two different locations (electrodes labeled ' 1 ' and '2') and at the axon (top), and corresponding activation curves (bottom).

\section{Electrical activation threshold is inversely related to recorded spike amplitude}

The relationship between El amplitude and ERF strength observed on axonal electrodes (Fig. 1, marker size and color) was consistent across cells. Recording and stimulation was examined on 61 electrodes overlying the axons of $53 \mathrm{ON}$ and OFF parasol cells. Axonal thresholds exhibited a clear inverse relationship to $\mathrm{El}$ amplitudes (Fig. 2 A\&B, red markers). To determine whether this relationship holds across preparations, recording and stimulation features from 152 axonal electrodes overlying 102 cells from 23 retinas were compared (Fig. 2C). Surprisingly, across multiple recordings, a consistent inverse relationship was observed for electrodes overlying axons of both ON and OFF parasol cells (Fig. 2C, circle and square markers respectively), similar to the trend observed in a single retina (Fig. $2 \mathrm{~A} \& \mathrm{~B}$, red points vs. points in $\mathrm{C}$ ). The relationship between axonal $\mathrm{El}$ amplitudes and activation thresholds was fitted by an inverse function chosen based on studies indicating that electrode distance is linearly related to activation threshold for electrodes less than $50 \mu \mathrm{m}$ away (Rattay and Wenger, 2010): threshold $=\frac{a}{\text { amplitude }-c}+b$, with maximum likelihood fit parameters $a=6.5, b=0.4 \mu \mathrm{A}$, and $c=35$ (Fig 2C, dashed line, see Methods).

Similarly to axonal activation thresholds, somatic thresholds exhibited an inverse dependency on El amplitude, as observed in 55 somatic electrodes overlying $53 \mathrm{ON}$ and OFF parasol cells in two different retinal preparations (Fig. 2 A\&B, blue markers). However, pooling the recording and stimulation features, and inspecting the locations of 94 somatic electrodes overlying 102 cells across 23 retinas revealed that somatic thresholds depended on the distance of the stimulating electrode from the putative location of the AIS (Fig. 2D, marker colors; see Methods). Specifically, for a given RGC, somatic electrodes near the AIS (Fig. 2D, blue markers below dashed line) required less current to cause activation than electrodes farther away (Fig. 2D, red markers above dashed line), despite recording spikes with similar peak amplitudes. Quantitative characterization of this trend revealed that the fractional difference between measured somatic activation thresholds and estimates based solely on El amplitude increased roughly linearly as a function of electrode distance from the AIS $\left(r^{2}=0.78\right)$. Taken together, these findings suggested the following equation for estimating somatic activation thresholds from recorded somatic spikes:

threshold $_{\text {soma }}=\left(\frac{a}{\text { amplitude-c }}+b\right) *\left(1+d *\right.$ distance $\left._{\text {ais }}+e\right)$, where after fitting, $\mathrm{a}=22, \mathrm{~b}=0.4 \mu \mathrm{A}, \mathrm{c}=96$ $\mu \mathrm{V}, \mathrm{d}=0.0146$, and $\mathrm{e}=-0.428$. Note that axonal electrodes did not exhibit any discernible dependence on distance to the AIS (Fig. 2C, marker colors) or to the midline of the axon (see Methods).

Across many cells, electrodes near dendrites did not reliably elicit spikes (Fig 1A bottom, black trace). On average, only $9 \%$ of identified electrodes recording significant spikes (see Methods) from each cell were identified as recording from dendrites (3577 cells, 34 retinas). Of these electrodes, none were able to elicit spikes with probability greater than 0.5 over the range of amplitudes tested, compared to $56 \%$ for all axonal electrodes and $72 \%$ for all somatic electrodes. Thus, the dendritic data were insufficient to explore the EI amplitude-activation threshold relationship and were not further analyzed. 

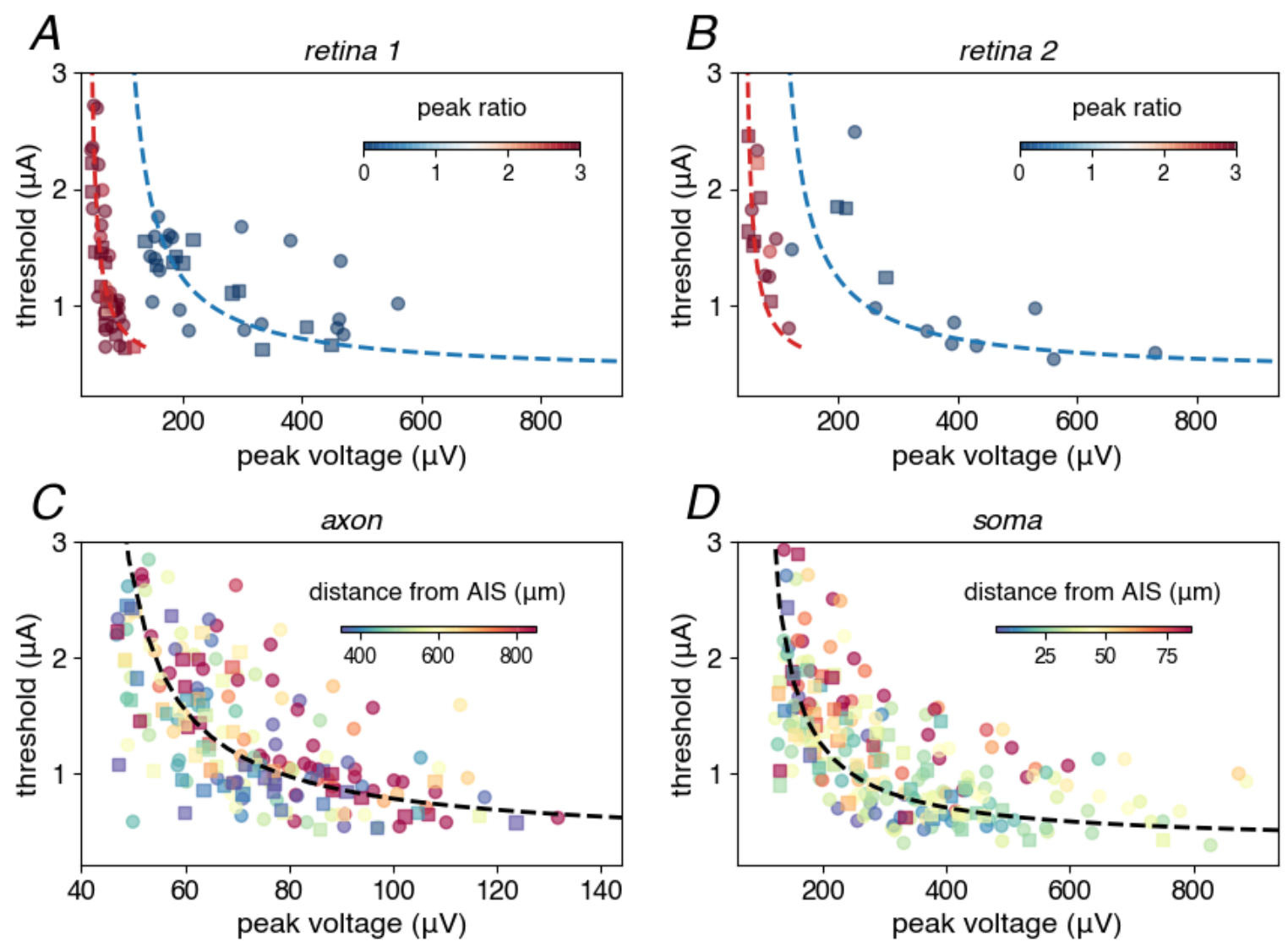

Figure 2. Relationship between activation thresholds and El amplitudes. In all plots, points corresponding to ON Parasol cells are denoted by circular markers, and OFF Parasol cells by square markers. A\&B) Electrical activation threshold vs. recorded spike amplitude for axonal (red markers) and somatic (blue markers) electrodes in two different retinal preparations; marker color corresponds to the ratio of the positive peaks of the recorded waveform on each electrode. Respectively colored dashed lines correspond to maximum log-likelihood, first-order inverse curve fits to the aggregated data for somas (blue, fit to data in C) and axons (red, fit to data in D). Aggregated thresholds vs. El amplitudes for axonal (C) and somatic (D) electrodes, collected from 23 different retinas; dashed lines correspond to maximum log-likelihood, first-order inverse curve fits to the data. Marker color corresponds to distance from the axon initial segment (AIS) location.

\section{Activation curve slope is inversely related to activation threshold}

A complete characterization of RGC responses to electrical stimulation entails determining not only the $50 \%$ activation threshold, but also the spiking probability over a range of stimulus amplitudes--i.e. a full activation curve (Fig. 3A). A simple possibility is that, for each cellular compartment, the activation curve has a stereotyped form across cells, because of the similarities in morphology and membrane dynamics between cells, and that this form scales with the injected current according to the electrical impedance between the electrode and the site of activation. In this case, normalizing measured activation curves by their associated threshold should result in a constant form. Indeed, this normalization revealed that activation curves are strikingly similar across retinal preparations for all somatic electrodes, and for all axonal electrodes, but very different between the two compartments (Fig 3C, blue vs. red curves). Axonal activation curves featured a relatively steep, "all-or-none" relationship, while somatic activation curves were shallower.

The similarity of normalized activation curves within each cellular compartment indicates that the slope is inversely related to the threshold. To characterize this relationship further, activation curve slopes were examined as a function of activation threshold, separately for 94 somatic and 152 axonal electrodes (Fig. 3 
B\&D). This relationship was fitted by an inverse function: slope $=\frac{a}{(\text { threshold }-c)}+b$. Maximum likelihood fits to the data resulted in $a=3.94$ for somas and 4.91 for axons, $b=2.25$ for somas and 8.72 for axons, and $c=$ 0.19 for somas and 0.6 for axons (Fig. 3 B\&D, dashed lines). For each compartment, ON and OFF parasol cells exhibited a similar relationship between activation curve slope and threshold (Fig. 3 B\&D, circular and square markers, respectively). The high fidelity of these relations $\left(r^{2}=0.82\right.$ for somas and 0.74 for axons) suggests that characterizing the ERF of a cell (which only reflects threshold at each electrode) is sufficient to reconstruct the full set of activation curves for the cell at each electrode.
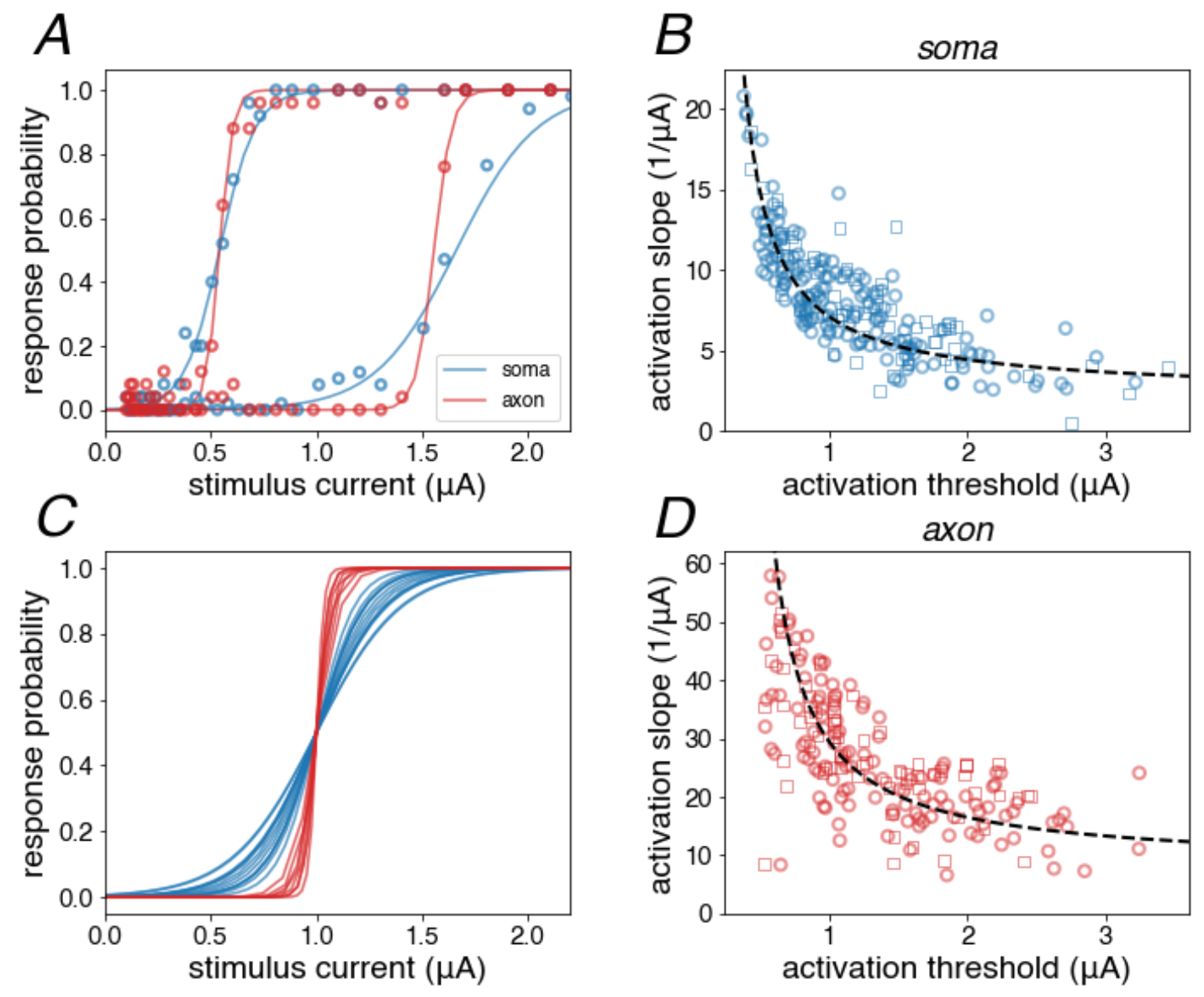

Figure 3. Relationship between activation curve slopes and activation thresholds. A) Four example somatic (blue) and axonal (red) measured sigmoidal activation curves. Measured spiking data is shown for one axonal and somatic electrode each (red \& blue circles). C) Activation curves shown in (A) along with 10 additional examples for each compartment, after current values were normalized by each curve's activation threshold. Activation curve slopes vs. thresholds for somatic (B) and axonal (D) electrodes, with first-order inverse curve fits (black, dashed line). Fitted activation curves with spuriously high slopes resulting from poorly estimated spiking probabilities were excluded from the figure (see Methods).

\section{Activation curves can be accurately inferred from electrical images}

The systematic relationship between El amplitude and activation probability probed above could be used to infer the parameters of ON and OFF parasol cell activation curves over space, using only recorded spikes, potentially conferring an advantage over naively averaging previously measured activation data. To test this possibility, the previously fitted inverse relations were used to estimate thresholds, which were then compared to measured thresholds, for the somas and axons of 102 cells (Fig. 4 A, example ON Parasol cell), across 246 electrodes from 23 different preparations (Fig. 4 B,C,E,F). The accuracy of inferred thresholds was compared 
to that of a single, naive estimate of the activation threshold obtained by averaging measured activation curve parameters over axonal and somatic electrodes across 53 retinal preparations (Fig. 4 B,C,E,F horizontal dashed lines, average threshold $1.2 \mu \mathrm{A}$, average slope $12.11 / \mu \mathrm{A})$. Inferred ERFs were qualitatively similar to measured ERFs for individual cells (Fig. 4A left vs. right, circle colors). Across axonal electrodes, $56 \%$ of activation thresholds were estimated within $25 \%$ of the measured value, compared to $33 \%$ obtained by averaging (Fig. 4E). Across somatic electrodes, $48 \%$ of inferred thresholds were within $25 \%$ of the measured value, compared to $28 \%$ obtained by averaging (Fig. $4 \mathrm{~B}$ ). Activation slopes were inferred from these estimated activation thresholds, and were within $25 \%$ of the measured slope value for $58 \%$ of axonal electrodes, compared to $14 \%$ using the average slope (Fig. 4F), and $67 \%$ of somatic electrodes, compared to $22 \%$ using the average slope (Fig. 4C).

Complete activation curves inferred using features of the El can then be used to estimate activation probabilities over the range of applied stimulus current levels, more effectively than the naive estimate derived by averaging. This was determined by pooling data across 246 electrodes and comparing measured curves to the corresponding inferred curves (using the fitted inverse relations for threshold and slope inference, respectively), or to a single, naively estimated sigmoidal activation curve derived from average parameters (same as above). The difference between each measured and estimated sigmoidal activation curve was estimated by computing the absolute difference of the integrated area between the curves. The separation between fitted and inferred activation curves was significantly smaller than the separation between fitted and fixed curves for somas and axons (Fig 4. D\&G, Wilcoxon signed rank test between green and purple histograms: $p=0.002$ for somas, $p=0.02$ for axons). The mean separation for inferred somatic activation curves was $22 \%$ closer to zero than for the fixed curve, and $30 \%$ closer for axons (Fig. 4 D\&G, vertical dashed lines).

A
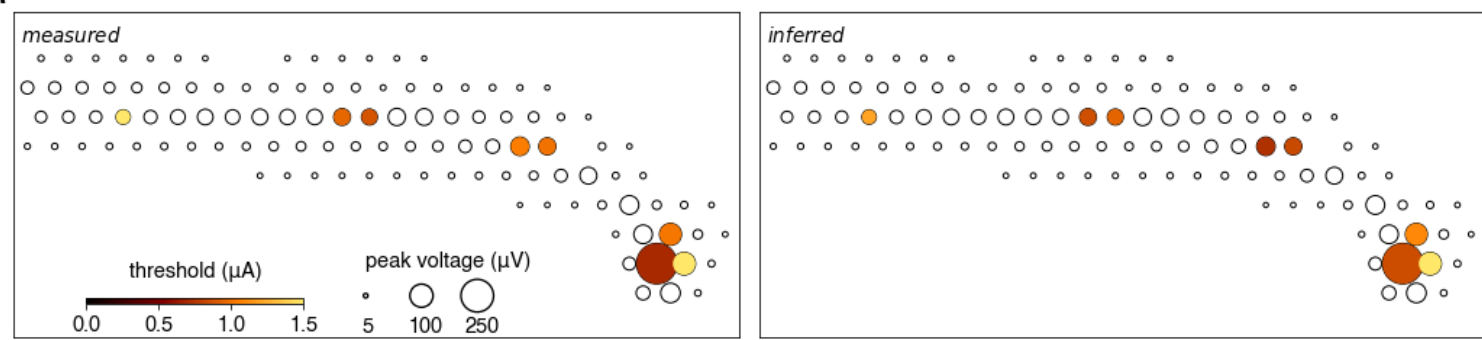

$B$

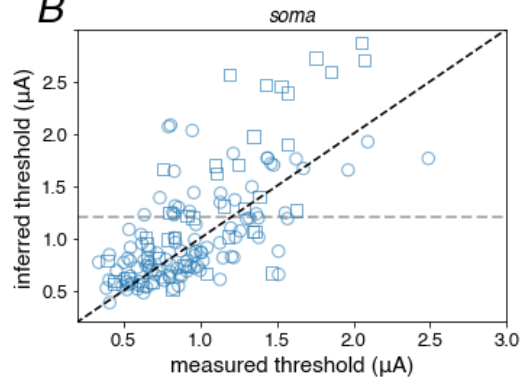

E axon

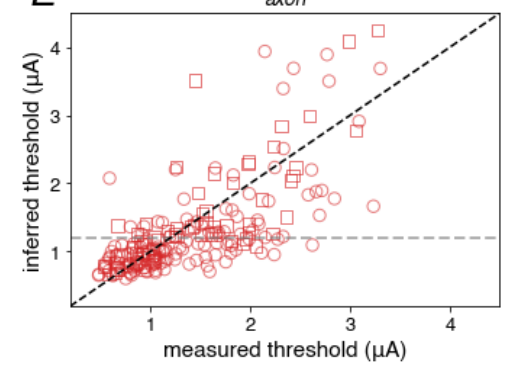

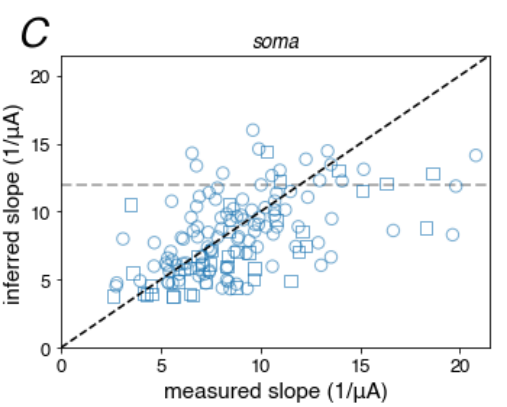
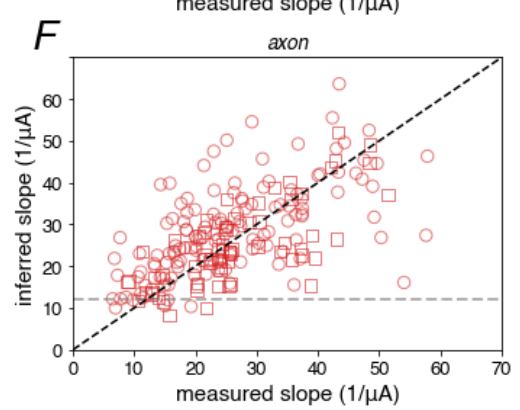
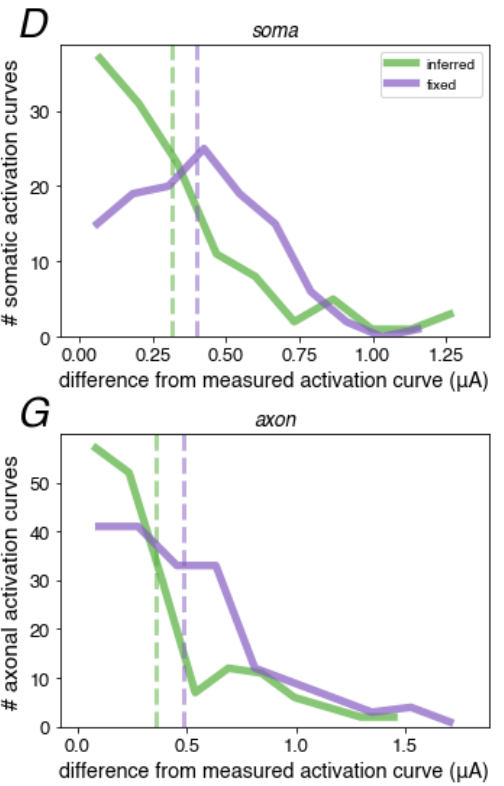
Figure 4. Comparison of inferred and measured activation properties. A) Measured vs. inferred ERFs for a single ON Parasol. Circle size corresponds to spike amplitude recorded at each El electrode; circle shade corresponds to measured or inferred thresholds at each electrode. Inferred vs. measured somatic $(B)$ and axonal $(E)$ thresholds, and slopes ( $\&$ \&), pooled across many electrodes. ON and OFF parasols are indicated by circular and square markers. Dashed line corresponds to $x=y$. D) Outlined histograms of the absolute differences of integrals between fitted and inferred (green), or fixed (purple), somatic activation curves, with respective mean values indicated by vertical dashed lines. G) Absolute differences of integrals for axonal activation curves.

\section{Biophysical simulations corroborate experimentally observed extracellular activation trends}

Simulated extracellular stimulation of a mammalian RGC (Fohlmeister and Miller, 1997) reproduced the main empirically observed trends in electrical activation properties. Simulations were conducted as follows (see Methods). The NEURON software package (Carnevale and Hines, 2006) was used to implement a previously published five-channel electrical circuit model of a rat RGC, with slightly simpler axonal geometry based on morphological measurements from macaque RGCs. The model cell was simulated in a uniform medium with isotropic resistivity. To simulate nondeterministic activation, spontaneous membrane voltage fluctuations (arising from sources such as channel noise and synaptic inputs) were modeled as Gaussian noise with a standard deviation of $2 \mathrm{mV}$ in each model segment (see Methods; F. Rieke, personal communication). Stimulating electrodes were modeled as a row of point current sources with $60 \mu \mathrm{m}$ spacing along the cell and axon, at a retinal depth $20 \mu \mathrm{m}$ from that of the axon (Fig. 5A, black, blue, and red row of bars spanning the top). Tissue impedances were fixed in the simulation, except at two locations corresponding to the soma and distal axon (Fig. 5A, blue and red bars) where impedance was varied $\pm 75 \%$. Voltages at each simulated electrode were obtained by summing the voltage fluctuations resulting from action potentials in the various model segments.

Analysis of these simulations corroborated several of the key trends described above in the recorded data. First, electrodes located over the soma recorded spikes with 3-5 times the amplitude of electrodes overlying axons, but activated the cell with similar current thresholds (Fig. 5C different circle sizes with similar colors). Second, electrodes recording somatic spike waveforms closer to the AIS had lower activation thresholds than electrodes farther from the AIS (Fig. 5C, leftmost circle colors). Third, stimulation and recording with somatic and axonal electrodes over a range of assumed tissue impedance values resulted in an inverse relationship between spike amplitude and activation threshold for both soma and axon electrodes, with the somatic curve displaced toward larger spike amplitudes (Fig. 5B, blue vs. red dots, and dotted vs. dashed curves). Finally, stimulation with increasing current produced steeper activation curves at axonal electrodes than at somatic electrodes (Fig. 5D, blue and red sigmoid curves). Taken together, these results suggest that well-understood, biophysical mechanisms underlie the experimentally observed relationships between electrical recording and activation of parasol cells presented above. 
$A$

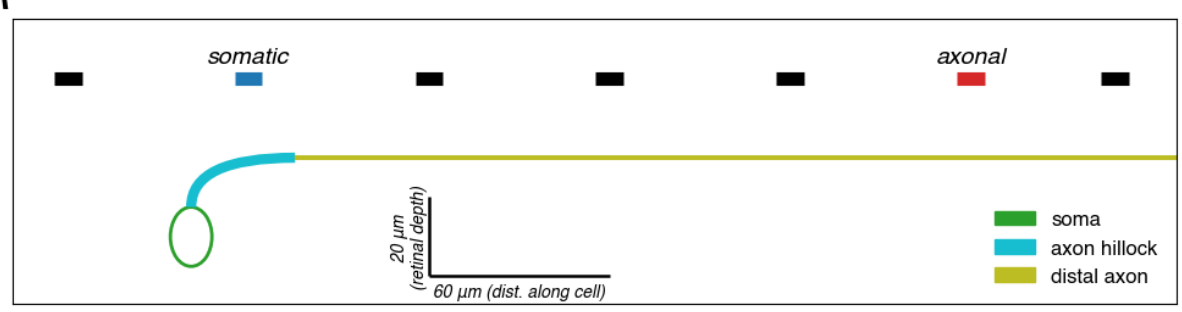

C

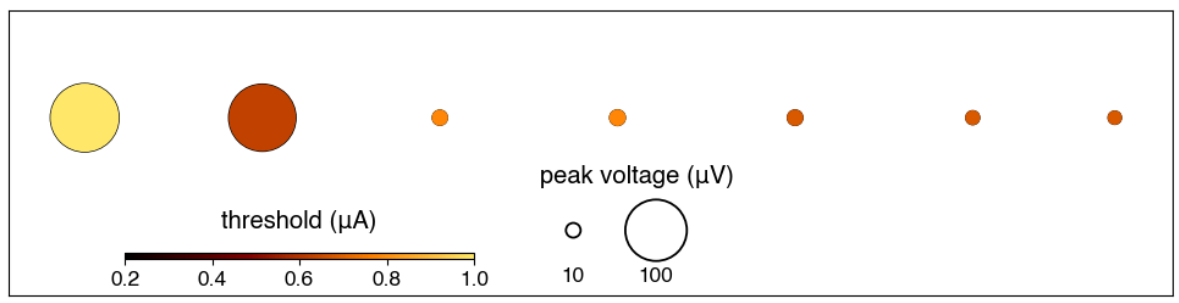

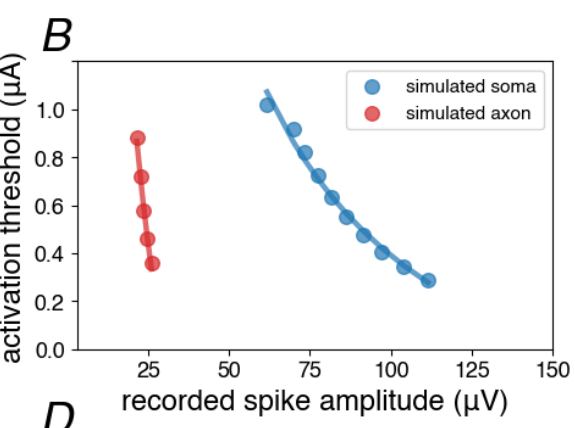

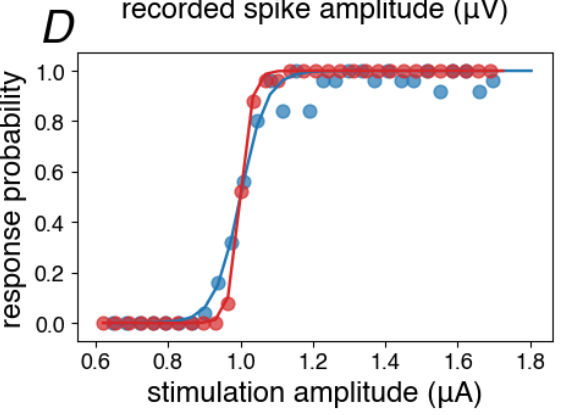

Figure 5. Extracellular activation properties of simulated RGCs. A) Finite-element model schematic showing soma, axon hillock, and distal axon segments, and overlying simulated electrode positions. B) Activation thresholds vs. recorded spike amplitudes for simulated (dots) and experimentally collected (dashed lines) data, for somatic (blue) and axonal (red) electrodes. C) Simulated ERF with electrode locations corresponding to each electrode $x$-position in (A). Circle sizes correspond to peak recorded spike amplitudes, colors indicate activation thresholds. D) Simulated somatic (blue) and axonal (red) response probability vs. stimulating current data fit by sigmoidal curves.

Inferred activation curves can be used to inform stimulation choices in a retinal implant

The information inferred about electrical activation over space was useful when tested in an electrical stimulation procedure (N. P. Shah et al., 2019) that could be potentially deployed in a high-resolution epiretinal implant. This was demonstrated by estimating the cumulative visual perception (or image reconstruction, (Brackbill et al., 2020; Warland et al., 1997)) resulting from a collection of current pulses delivered by electrodes across the array (see Methods), separately in three different retinal preparations. In each preparation, activation of each ON or OFF parasol cell was assumed to contribute a particular image component (or phosphene) to visual perception, corresponding to the optimal linear reconstruction filter obtained by estimating that cell's response to white-noise visual stimuli (see Methods), scaled by the spiking probability at the stimulating current level. Spiking probabilities were computed using either measured responses to electrical stimulation, inferred responses based purely on electrical recordings, or a fixed activation curve representing the average across 246 electrodes and 102 cells in 53 preparations (as in Fig. 4). Each image was translated into a spatial pattern of stimulation, using 40 individual electrodes and current amplitudes selected to minimize the normalized, pixel-wise mean-squared-error (NMSE, see Methods) between the reconstructed and true image.

Qualitative comparison between reconstructed images revealed that choosing electrodes based on inferred activation curves resulted in substantially more accurate reconstruction than could be obtained by using the fixed curve, and approached the best achievable reconstruction obtained using measured activation curves (Fig. 6A, example reconstructions of two target images in a single preparation). Comparing the accuracies of 45 reconstructed targets across 3 retinal preparations revealed that choosing stimuli using inferred activation curves provided a 54\% improvement in NMSE over using fixed curves (see Methods, Fig. 6B, dashed red, 
green, and black dashed line slopes). These results suggested that inference of activation curves from Els has a significant potential to produce more accurate visual perception with a future, closed-loop epiretinal implant.
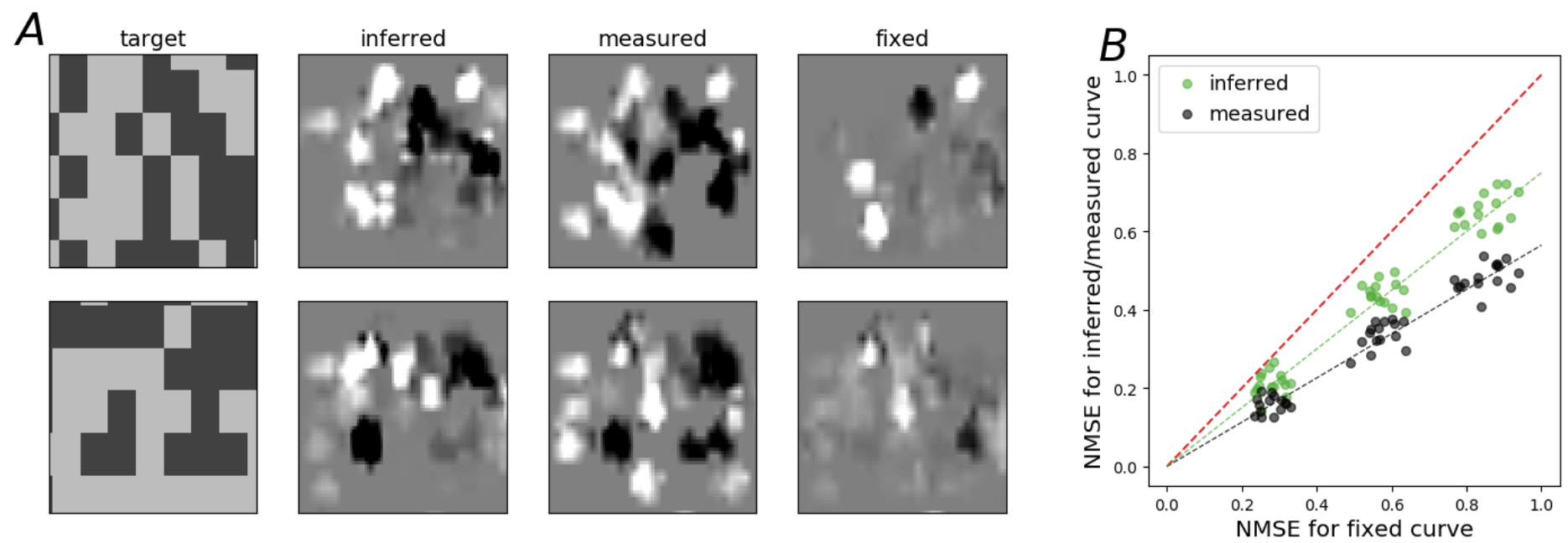

Figure 6. Simulated image reconstruction using inferred, measured, and fixed activation curves. A) Reconstructed images in one retinal preparation for two targets (top and bottom rows respectively, left), using inferred (middle-left), measured (middle), and fixed control (middle-right), activation curves. B) Normalized mean-squared-error (NMSE) for fixed vs. inferred (green markers) and measured (black markers) across 15 targets and 3 retinal preparations, corresponding to the three clusters of scattered points. Red dashed line corresponds to $\mathrm{x}=\mathrm{y}$, green dashed line represents linear fit to inferred scatter points, black dashed line represents linear fit to measured scatter points.

\section{Discussion}

The present results demonstrated that the effectiveness of electrical stimulation at a given electrode positioned near a RGC is inversely related to the recorded spike amplitude at that electrode, in a manner that is distinct for each cellular compartment, and consistent across many cells within and across retinas. These trends were qualitatively replicated in simulations with a biophysical model, suggesting that they arise from well-understood properties of neurons. The observed relationships can be harnessed to accurately infer the extracellular activation properties of cells over space based solely on recording natural activity, and thus may be useful in guiding electrical stimulation choices relevant to prosthetic vision.

Several key features of electrical activation properties presented here are supported by previous studies. First, the inverse relationship between spike amplitude and activation threshold agrees with theoretical predictions of the distance-threshold relationship for extracellular stimulation. These predictions are based on the assumption that extracellular activation depends on the second spatial derivative of the voltage deflection along the membrane of the axon (Rattay, 1987; Rattay and Wenger, 2010), and suggest that at electrode distances less than $\sim 50 \mu \mathrm{m}$, the distance-threshold relationship should be linear. The linear relationship in turn supports the observed inverse relationship between recorded spike amplitude and threshold. In addition, reports from previous experimental and theoretical studies indicate that the AIS, which is positioned between the soma and distal axon of RGCs, is highly excitable (Boiko et al., 2003; Esler et al., 2018b; Fried et al., 2009; Radivojevic et al., 2016; Rattay and Wenger, 2010; Werginz et al., 2020), consistent with the observed increase in activation threshold with increasing distance from the AIS for electrodes near the soma. On the other hand, it is important to note that applying the inference relations presented in this work to other devices may necessitate the collection of at least some electrical stimulation data in the target retina, because stimulation thresholds (but 
not recorded spike amplitudes) may vary based on electrode geometry (Cao et al., 2015; Lempka et al., 2011; Viswam et al., 2019).

In a notable previous study, inference of activation thresholds from the recorded features of electrically evoked spikes was performed in 14 cultured cortical neurons (Radivojevic et al., 2016). Inference was performed using multivariate regression on eight features of recorded spikes, revealing that the two critical features for inference were spike amplitude and time of spike onset. These two features roughly correspond to those used for threshold inference in the present work: spike onset times often identify the cellular compartment, due to the stereotyped time course of action potential propagation.

Several limitations of the present work bear mentioning. First, certain cell-electrode pairs could not be analyzed reliably. Specifically, electrodes recording spike amplitudes smaller than twice the standard deviation $(\sim 15-20$ $\mu \mathrm{V}$ ) of the recording noise were not analyzed, due to the difficulty of accurately identifying low-amplitude spike waveforms in the post-stimulation voltage traces. This led to the exclusion of parasol cells with small spikes, as well as midget cells, the numerically dominant RGC class with greatest relevance to high-acuity vision.

Second, the results were obtained from healthy primate retinas; degenerate retinas would be more relevant for application to epiretinal implants. Experimental and computational studies of electrical stimulation using rat models of retinal degeneration indicate that recorded spike waveforms and stimulation thresholds do not significantly change with degeneration (Loizos et al., 2018; Sekirnjak et al., 2009). However, other studies employing stimulation using long pulses and large electrodes in mouse models suggest that thresholds increase (Jensen and Rizzo, 2008; O'Hearn et al., 2006; Suzuki et al., 2004). It remains to be seen whether the relationship between recorded spikes and activation thresholds is the same in diseased and healthy primate retina. Additionally, the present work was also performed entirely in the peripheral retina, whereas a clinical implant would likely be placed in the central retina.

Finally, the biophysical model (Fohlmeister et al., 2010; Fohlmeister and Miller, 1997) captured general trends in the data, but did not quantitatively reproduce the experimental results. This discrepancy may reflect several factors. The properties of the model cell are derived from rat ON-alpha RGCs, which may differ from primate parasol RGCs in crucial features such as cell size and channel density, and were obtained at room temperature $\left(22^{\circ} \mathrm{C}\right)$, which produces slower channel kinetics than would be observed at physiological temperatures. The model also does not distinguish between the various subtypes of sodium channels in distinct cellular compartments (Boiko et al., 2003; Van Wart and Matthews, 2006). Additionally, the resistivities of the retinal layers in primates is unknown, with reported measurements varying significantly (Abramian et al., 2015; Kasi et al., 2019; Wang and Weiland, 2015). Lastly, modeling the non-deterministic nature of extracellular activation, which presumably results from membrane voltage fluctuations caused by ion channels and synaptic inputs, relies on patch clamp data that can be obtained reliably from RGC somas but not from axons. For these reasons, the model was used only to confirm general trends observed in the empirical data.

Despite these limitations, inference of RGC activation properties purely from spontaneous activity has the potential to improve the function of a future retinal implant, by wholly or partially eliminating the need to perform calibration of a large-scale retinal interface. This is important because electrical stimulus calibration generally requires sequential testing of each individual electrode, which is time-consuming, and more importantly is greatly complicated by electrical artifacts, which make it difficult to identify spikes evoked by electrical stimulation. These requirements could pose substantial technical challenges in the clinical setting, and highlight the value of the inference approach described. A hybrid approach could entail performing full electrical calibration only for cells with small recorded spikes, to supplement the more reliable inferred electrical 
activation curves of cells with large recorded spikes. Alternatively, initial estimates of electrical thresholds obtained from recording alone, or using inferred sensitivity as priors in Bayesian estimation (N. Shah et al., 2019), could be used to more quickly identify thresholds during full electrical stimulus calibration. Inference also allows for rapid re-calibration of electrical stimulation over time, potentially compensating for small movements of the implanted device. Furthermore, the similarity between the electrical impedance and neuronal activation characteristics of the retina and other neural circuits (Branner et al., 2001; Hillman et al., 2003; Rattay, 1987; Royeck et al., 2008) suggests that the ability to infer electrical activation properties from extracellular recordings could have widespread application for implanted devices in the brain.

Acknowledgements. We thank J. Carmena, T. Moore, W. Newsome, M. Taffe, S. Morairty, J. Horton, and the California National Primate Research Center for providing macaque retinas. We thank K. Jensen, M. Allen, and K. Tinajero, K. Berry, K. Williams, B. Morsey, J. Frohlich, and M. Kitano for helping obtain access to macaque retinas and metadata. We thank D. Palanker, F. Rieke, S. Mitra, P. Li, T. Carnevale, M. Hines, F. Rattay, P. Tandon, E. Wu, R. Vilkhu, V. Fan, M. Zaidi, C. Rhoades, N. Brackbill, G. Goetz, S. Wienbar, and the Stanford Artificial Retina team for helpful discussions. We thank R. Samarakoon and S. Kachiguine for technical assistance. We thank L. Jepson, P. Li, M. Greshner, G. Field, J. Gautier, A. Heitman, and G. Goetz for participating in data collection. Support: NIH NEI F30-EY030776-03 (SM), Polish National Science Centre grant DEC-2013/10/M/NZ4/00268 (PH), Pew Charitable Trust Scholarship in the Biomedical Sciences (AS), a donation from John Chen (AML), Research to Prevent Blindness Stein Innovation Award, Wu Tsai Neurosciences Institute Big Ideas, NIH NEI R01-EY021271, NIH NEI R01-EY029247, and NIH NEI P30-EY019005 (EJC).

Author Contributions. Conceptualization, S.S.M., L.E.G., and E.J.C.; Methodology, S.S.M., L.E.G., N.P.S., and E.J.C.; Investigation, S.S.M., L.E.G., N.P.S., A.K., and A.R.G.; Writing - Original Draft, S.S.M. and E.J.C.; Writing - Review \& Editing, S.S.M. and E.J.C.; Funding Acquisition, S.S.M., P.H., A.S., A.M.L., and E.J.C.; Resources, S.S.M., L.E.G., N.P.S., A.K., A.R.G., P.H., A.S., A.M.L., and E.J.C.; Supervision, E.J.C.

\section{Declaration of Interests}

The authors declare no competing interests.

\section{Methods}

\section{Experimental setup}

A custom 512-electrode system (Grosberg et al., 2017; Hottowy et al., 2012, 2008) was used to stimulate and record from retinal ganglion cells (RGCs) in isolated rhesus macaque monkey (Macaca mulatta) retinas. Retinas were obtained from terminally anesthetized animals euthanized during the course of research performed by other laboratories, in accordance with institutional and national guidelines and regulations. Ocular hemisection was performed in ambient indoor lighting following enucleation, the vitreous was removed, and the posterior portion of the eye was stored in warm, oxygenated, bicarbonate buffered Ames' solution (Sigma-Aldrich) in darkness. Patches of retina $\sim 3 \mathrm{~mm}$ on a side were isolated under infrared light, placed RGC side down on the multielectrode array, and superfused with Ames solution at $35^{\circ} \mathrm{C}$. Electrodes were $8-15 \mu \mathrm{m}$ in diameter and were electroplated with platinum. The multi-electrode array (MEA) consisted of 512 electrodes arranged in a $16 \times 32$ isosceles triangular lattice with $60 \mu \mathrm{m}$ spacing (Litke et al., 2004). Voltage recordings were band-pass filtered between 43 and $5,000 \mathrm{~Hz}$ and sampled at $20 \mathrm{kHz}$. Spikes in the voltage recordings from individual RGCs evoked by light stimulation (which produced no electrical artifacts) were identified and sorted using previously described spike sorting techniques (Litke et al., 2004). 
To identify the type of each recorded cell, as well as the location and shape of the visual receptive field (RF), the retina was visually stimulated with a dynamic, binary white noise stimulus, and the spike-triggered average (STA) stimulus was computed for each RGC (Chichilnisky, 2001; Chichilnisky and Kalmar, 2002). The STA summarizes the spatial, temporal, and chromatic structure of the light response. The spatial RFs and time courses obtained from the STA were used to identify the distinct cell types, as described previously (Chichilnisky and Kalmar, 2002; Field et al., 2007; Rhoades et al., 2019). Analyses in this study were limited to ON and OFF parasol RGCs, because spikes recorded from those cell types had high amplitudes that allowed for relatively fast, reliable electrical spike sorting (see below).

\section{Electrical image (El) computation}

An electrical signature for each neuron on the array was obtained by computing the electrical image (or $\mathrm{EI}$ ) (Litke et al., 2004). The El of each cell (e.g. Fig. 1A) represents the average spatiotemporal pattern of voltage deflections produced on each electrode of the array during a spike from a given cell. A minority $(\sim 3 \%)$ of spike clusters identified during spike sorting erroneously merged two different cells, and were excluded from analysis based on visual inspection. The remaining cells were used for subsequent analyses if their Els featured spike amplitudes greater than twice the standard deviation of the recorded voltage trace (i.e. the electrical recording noise) at a minimum of three recording electrodes.

\section{Identifying cellular compartments and landmarks}

As observed previously (Litke et al., 2004; Müller et al., 2015), the biophysical properties of somas, axons, and dendrites cause the spike waveforms recorded on MEA electrodes overlying each subcellular compartment to have distinct shapes (Fig. 1A, top row). Compartments were identified at each El electrode in two steps. First, waveforms with a larger positive phase than negative phase were labeled as dendrites. Second, the waveforms at remaining electrodes were identified as somatic, mixed, or axonal, based on the ratio of the first positive peak to the second positive peak. Thresholds for each respective compartment were determined by two empirically observed inflection points ( 0.06 and 1.32 respectively) in the cumulative distribution of ratios-across tens of thousands of spike waveforms obtained from thousands of recorded parasol cells.

The identification of the cellular compartment at each electrode allowed for the estimation of two morphological landmarks for each RGC used in several analyses. The location of each RGC soma was estimated by taking the centroid of all identified somatic electrodes, weighted by the negative peak amplitude recorded on each electrode. The orientation of the axon was determined by computing a vector from the soma centroid to the nearest axonal electrode more than $180 \mu \mathrm{m}$ away. The axonal-initial-segment (AIS) location was assumed to be $13 \mu \mathrm{m}$ along this vector from the soma centroid (Sekirnjak et al., 2008). The axon midline was estimated by conducting amplitude-weighted fitting of a 3rd degree polynomial spline to all identified axonal electrodes.

\section{Electrical stimulation and spike sorting}

Electrical stimuli consisted of triphasic current waveforms consisting of a cathodal phase flanked by two anodal charge-balancing pulses, with relative amplitudes 2:-3:1. Each phase was $50 \mu$ in duration. The shape of the triphasic pulse was chosen to minimize the recorded electrical stimulation artifact (Hottowy et al., 2012). At each of the 512 electrodes on the MEA, triphasic pulses with thirty-nine stimulating-phase amplitudes increasing from 0.1 to $4.1 \mu \mathrm{A}$ in $10 \%$ increments were delivered 25 to 50 times each. Spikes were elicited with sub-millisecond temporal precision (Jepson et al., 2013) by directly depolarizing the cell, as shown by synaptic transmission blockade (Sekirnjak et al., 2006). A semi-automated method was used to subtract electrical 
artifacts from the raw post-stimulation data and assign spikes to cells using waveform templates derived from their Els, as described previously (Jepson et al., 2013; Mena et al., 2017). To enable accurate spike assignment, at each electrode, only cells with recorded spike amplitudes of at least $50 \mu \mathrm{V}$ were analyzed, because spikes with lower amplitudes were difficult to distinguish from background electrode noise. Stimulation amplitudes above axon bundle activation thresholds, which were estimated at each electrode using a previously described method (Tandon et al., 2021), were not analyzed in order to avoid potential off-array RGC activation. For each stimulus amplitude, the evoked spike probability was computed across repeats. The dependence of spike probability on stimulation current amplitude was modeled by a sigmoidal relationship:

$$
p(a)=\frac{1}{1+e^{-k(a-b)}}
$$

where $a$ is the current amplitude, $p(a)$ is the spike probability, and $k$ and $b$ are free parameters. Fitted sigmoidal curves were used to compute the activation threshold (b), defined as the current amplitude that elicits a spike with $50 \%$ probability, and activation slope (k). All fitted curves were manually inspected for goodness of fit, and a small minority $(\sim 5 \%)$ were discarded due to either overfitting on spuriously identified spikes, or mistaken assignment of spikes to neighboring cells.

\section{Retinal preparation, cell, and activation curve selection}

Of 53 total preparations from 50 different macaque retinas, 23 were included. In these preparations, the excised segment of retina covered more than $80 \%$ of the electrodes on the array, and the population RGC firing rate exhibited variance less than $20 \%$ of the mean. Only somatic and axonal cell-electrode pairs recording spikes greater than $50 \mu \mathrm{V}$, with activation curve thresholds less than both the bundle threshold on that electrode, which accounted for $\sim 80 \%$ of electrodes unable to be analyzed, and the upper current limit of the stimulation range $(4 \mu \mathrm{V})$ were considered for analysis. Based on these criteria, 246 cell-electrode pairs from 59 ON and 43 OFF parasol cells were analyzed.

\section{Derivation and fitting of inference relations}

The relationship between activation threshold and recorded spike amplitude, and the relationship between activation curve slope and threshold, were both modeled as inverse functions of the form $f(x)=\frac{a}{(x-c)}+b$. This functional form was chosen based on theoretical predictions from the literature (Rattay and Wenger, 2010), and the observation that normalizing sigmoidal activation curves by their thresholds resulted in curves with similar slopes within each cellular compartment. Inverse functions for each activation curve parameter were fitted by maximizing the log-likelihood of the evoked spike probability data.

\section{Simulated image reconstruction}

Simulation of perceived images resulting from application of stimulating current pulses, and selection of optimal electrical stimuli, were based on previous work (Shah et al., 2019) and consisted of several steps.

First, the contribution of RGC activity to visual perception was estimated in each of three retinal preparations, assuming that perception represents an optimal linear reconstruction of the stimulus from the RGC responses (Brackbill et al., 2020; Warland et al., 1997). Optimal linear reconstruction was examined by simulating the response of each cell to $10,00012 \times 6$ random black and white noise training images, based on the spatiotemporal STA (see above), and then computing reconstruction filters from the simulated responses for 
each cell as follows. The simulated nonnegative response to each training image was estimated by taking the inner product of the STA and that image, and rectifying the result. Then, the reconstruction filter was obtained by performing least squares regression of the simulated responses against the set of training stimuli.

Next, for each of 15 randomly generated $12 \times 6$ black and white images, 40 electrical stimuli were chosen to (approximately) produce an appropriate neural response in each retinal preparation. Each stimulus consisted of a particular current amplitude on a particular electrode and produced spikes with probabilities from 0 to 1 in one or more RGCs, estimated using either measured, inferred, or fixed activation curves. The fixed curve was assumed using parameters of $1.2 \mu \mathrm{A}$ for threshold and $12.1 \mu \mathrm{A}^{-1}$ for slope, obtained by averaging across all measured responses to electrical stimulation in 53 preparations (see Fig. 4 B-G). For each type of activation curve, stimuli were chosen so that the sum of their presumed contributions to perception, based on the linear reconstruction filters weighted by the expected response, minimized the normalized mean squared error (NMSE) between the stimulus and reconstructed image. Normalization was relative to the highest achievable mean-squared error across images and retinas, for ease of interpretation. The number of electrical stimuli (40) was empirically determined as the value beyond which, on average, reconstruction using inferred activation curves no longer reduced the NMSE.

To quantify the effectiveness of using inferred over fixed activation curves for image reconstruction, NMSE values of reconstructed images based on the three curve types were collected for each retinal preparation and target image. Then, NMSEs for inferred and measured activation curves were compared to NMSEs for fixed curves (see Fig. 6B). Across preparations and targets, systematic deviations from the $x=y$ line, which corresponds to using the fixed curve, were captured by slopes obtained from linear regression. Finally, the overall value of performing reconstruction with inferred activation curves was given by $\left|\left(s_{m}-s_{i}\right) /\left(s_{m}-1\right)\right|$, where $s_{m}$ is the slope obtained with measured activation curves and $s_{i}$ is the slope obtained with inference.

\section{Biophysical simulations}

RGC simulation was conducted using the NEURON software package (Hines and Carnevale, 2001) to create a model cell featuring five ion channel types, along with an extracellular stimulation mechanism, with an integration timestep of $0.001 \mathrm{~ms}$. The properties of the model RGC were based on ion channel densities and compartment sizes adapted from voltage-clamp and morphology measurements of rat ON-alpha RGCs (Fohlmeister et al., 1990; Fohlmeister and Miller, 1997). Slight modifications to the original model geometry were made to increase resemblance to macaque peripheral parasol RGCs, guided by previous measurements (Grosberg et al., 2017; Jeng et al., 2011; Peterson and Dacey, 1998; Watanabe and Rodieck, 1989). These included eliminating the axonal narrow segment, increasing the thickness of the axon, and introducing a bend in the axon hillock (see Fig. 5A). The final model consisted of a spherical soma with a diameter of $20 \mu \mathrm{m}$, an axon hillock with a diameter of $4.5 \mu \mathrm{m}$, and a distal axon with a diameter of $2 \mu \mathrm{m}$. For this simulation, the dendritic compartment was excluded.

The extracellular environment was assumed to be an isotropic, uniform medium with a linear resistivity of 0.5 kOhm-cm (Kasi et al., 2019). Extracellular stimulation was conducted with a collection of simulated point-electrodes located at a retinal depth of $20 \mu \mathrm{m}$ relative to the axon, at $60 \mu \mathrm{m}$ intervals along the length of the soma and axon (see Fig. 5A). Post-stimulation voltages resulting from simulated action potential currents in the model cell were measured at each electrode location. At one somatic and one axonal electrode, the baseline resistivity of the extracellular medium was varied from 125 to $875 \mathrm{kOhm}-\mathrm{cm}( \pm 75 \%$ of the baseline resistivity of the medium) to simulate various effective distances from the cell. Stimulus waveforms consisted of triphasic current pulses with shapes and amplitudes matching those used in ex vivo experiments (see above). 
The non-deterministic nature of extracellular stimulation putatively arises from stochastic membrane voltage fluctuations, caused by a combination of rapid ion channel state changes and synaptic inputs. To approximate these fluctuations, random voltages drawn from a Gaussian distribution with a mean of $0 \mathrm{mV}$ and a standard deviation of $2 \mathrm{mV}$, based on empirically observed electrical noise levels (F. Rieke, personal communication), were added to the membrane voltage of each model segment, at intervals of $0.1 \mathrm{~ms}$.

The robustness of qualitative trends resulting from simulation was confirmed by varying model parameters. lon channel densities and diameters were scaled $\pm 75 \%$ across all segments without affecting the direction of the trends. Varying the retinal depth of the electrodes from $10 \mu \mathrm{m}$ (closer than which stimulation was not possible) to $100 \mu \mathrm{m}$ also preserved trends. .

\section{References}

Abramian, M., Lovell, N.H., Morley, J.W., Suaning, G.J., Dokos, S., 2015. Activation and inhibition of retinal ganglion cells in response to epiretinal electrical stimulation: a computational modelling study. J. Neural Eng. 12, 016002.

Boiko, T., Van Wart, A., Caldwell, J.H., Levinson, S.R., Trimmer, J.S., Matthews, G., 2003. Functional specialization of the axon initial segment by isoform-specific sodium channel targeting. J. Neurosci. 23, 2306-2313.

Boinagrov, D., Loudin, J., Palanker, D., 2010. Strength-Duration Relationship for Extracellular Neural Stimulation: Numerical and Analytical Models. J. Neurophysiol. 104, 2236-2248.

Brackbill, N., Rhoades, C., Kling, A., Shah, N.P., Sher, A., Litke, A.M., Chichilnisky, E.J., 2020. Reconstruction of natural images from responses of primate retinal ganglion cells. Elife 9.

Branner, A., Stein, R.B., Normann, R.A., 2001. Selective stimulation of cat sciatic nerve using an array of varying-length microelectrodes. J. Neurophysiol. 85, 1585-1594.

Cao, X., Sui, X., Lyu, Q., Li, L., Chai, X., 2015. Effects of different three-dimensional electrodes on epiretinal electrical stimulation by modeling analysis. J. Neuroeng. Rehabil. 12, 73.

Carnevale, N.T., Hines, M.L., 2006. The NEURON Book. Cambridge University Press.

Chichilnisky, E.J., 2001. A simple white noise analysis of neuronal light responses. Network 12, $199-213$.

Chichilnisky, E.J., Kalmar, R.S., 2002. Functional asymmetries in ON and OFF ganglion cells of primate retina. J. Neurosci. 22, 2737-2747.

Dacey, D., 2004. 20 Origins of Perception: Retinal Ganglion Cell Diversity and the Creation of Parallel Visual Pathways. In: Gazzaniga, M.S. (Ed.), The Cognitive Neurosciences lii. MIT Press, p. 281.

Esler, T.B., Kerr, R.R., Tahayori, B., Grayden, D.B., Meffin, H., Burkitt, A.N., 2018a. Minimizing activation of overlying axons with epiretinal stimulation: The role of fiber orientation and electrode configuration. PLoS One 13, e0193598.

Esler, T.B., Maturana, M.I., Kerr, R.R., Grayden, D.B., Burkitt, A.N., Meffin, H., 2018b. Biophysical basis of the linear electrical receptive fields of retinal ganglion cells. J. Neural Eng. 15, 055001.

Field, G.D., Chichilnisky, E.J., 2007. Information processing in the primate retina: circuitry and coding. Annu. Rev. Neurosci. 30, 1-30.

Field, G.D., Sher, A., Gauthier, J.L., Greschner, M., Shlens, J., Litke, A.M., Chichilnisky, E.J., 2007. Spatial properties and functional organization of small bistratified ganglion cells in primate retina. J. Neurosci. 27, $13261-13272$.

Fohlmeister, J.F., Cohen, E.D., Newman, E.A., 2010. Mechanisms and distribution of ion channels in retinal ganglion cells: using temperature as an independent variable. J. Neurophysiol. 103, 1357-1374.

Fohlmeister, J.F., Coleman, P.A., Miller, R.F., 1990. Modeling the repetitive firing of retinal ganglion cells. Brain Res. 510, 343-345.

Fohlmeister, J.F., Miller, R.F., 1997. Mechanisms by which cell geometry controls repetitive impulse firing in 
retinal ganglion cells. J. Neurophysiol. 78, 1948-1964.

Fried, S.I., Lasker, A.C.W., Desai, N.J., Eddington, D.K., Rizzo, J.F., 3rd, 2009. Axonal sodium-channel bands shape the response to electric stimulation in retinal ganglion cells. J. Neurophysiol. 101, 1972-1987.

Grosberg, L.E., Ganesan, K., Goetz, G.A., Madugula, S.S., Bhaskhar, N., Fan, V., Li, P., Hottowy, P., Dabrowski, W., Sher, A., Litke, A.M., Mitra, S., Chichilnisky, E.J., 2017. Activation of ganglion cells and axon bundles using epiretinal electrical stimulation. J. Neurophysiol. 118, 1457-1471.

Hillman, T., Badi, A.N., Normann, R.A., Kertesz, T., Shelton, C., 2003. Cochlear nerve stimulation with a 3-dimensional penetrating electrode array. Otol. Neurotol. 24, 764-768.

Hines, M.L., Carnevale, N.T., 2001. NEURON: a tool for neuroscientists. Neuroscientist 7, 123-135.

Hottowy, P., Dąbrowski, W., Skoczeń, A., Wiącek, P., 2008. An integrated multichannel waveform generator for large-scale spatio-temporal stimulation of neural tissue. Analog Integr. Circuits Signal Process. 55, 239-248.

Hottowy, P., Skoczeń, A., Gunning, D.E., Kachiguine, S., Mathieson, K., Sher, A., Wiącek, P., Litke, A.M., Dąbrowski, W., 2012. Properties and application of a multichannel integrated circuit for low-artifact, patterned electrical stimulation of neural tissue. J. Neural Eng. 9, 066005.

Jeng, J., Tang, S., Molnar, A., Desai, N.J., Fried, S.I., 2011. The sodium channel band shapes the response to electric stimulation in retinal ganglion cells. J. Neural Eng. 8, 036022.

Jensen, R.J., Rizzo, J.F., 3rd, 2008. Activation of retinal ganglion cells in wild-type and rd1 mice through electrical stimulation of the retinal neural network. Vision Res. 48, 1562-1568.

Jepson, L.H., Hottowy, P., Mathieson, K., Gunning, D.E., Dabrowski, W., Litke, A.M., Chichilnisky, E.J., 2013. Focal electrical stimulation of major ganglion cell types in the primate retina for the design of visual prostheses. J. Neurosci. 33, 7194-7205.

Kasi, H., Meissner, R., Babalian, A., van Lintel, H., Bertsch, A., Renaud, P., 2019. Direct localised measurement of electrical resistivity profile in rat and embryonic chick retinas using a microprobe. J. electr. bioimpedance 1, 84-92.

Lempka, S.F., Johnson, M.D., Moffitt, M.A., Otto, K.J., Kipke, D.R., McIntyre, C.C., 2011. Theoretical analysis of intracortical microelectrode recordings. J. Neural Eng. 8, 045006.

Litke, A.M., Bezayiff, N., Chichilnisky, E.J., Cunningham, W., Dabrowski, W., Grillo, A.A., Grivich, M., Grybos, P., Hottowy, P., Kachiguine, S., Kalmar, R.S., Mathieson, K., Petrusca, D., Rahman, M., Sher, A., 2004. What does the eye tell the brain?: Development of a system for the large-scale recording of retinal output activity. IEEE Trans. Nucl. Sci. 51, 1434-1440.

Loizos, K., Lazzi, G., Lauritzen, J.S., Anderson, J., Jones, B.W., Marc, R., 2014. A multi-scale computational model for the study of retinal prosthetic stimulation. Conf. Proc. IEEE Eng. Med. Biol. Soc. 2014, 6100-6103.

Loizos, K., Marc, R., Humayun, M., Anderson, J.R., Jones, B.W., Lazzi, G., 2018. Increasing Electrical Stimulation Efficacy in Degenerated Retina: Stimulus Waveform Design in a Multiscale Computational Model. IEEE Trans. Neural Syst. Rehabil. Eng. 26, 1111-1120.

Mena, G.E., Grosberg, L.E., Madugula, S., Hottowy, P., Litke, A., Cunningham, J., Chichilnisky, E.J., Paninski, L., 2017. Electrical Stimulus Artifact Cancellation and Neural Spike Detection on Large Multi-Electrode Arrays.

Müller, J., Ballini, M., Livi, P., Chen, Y., Radivojevic, M., Shadmani, A., Viswam, V., Jones, I.L., Fiscella, M., Diggelmann, R., Stettler, A., Frey, U., Bakkum, D.J., Hierlemann, A., 2015. High-resolution CMOS MEA platform to study neurons at subcellular, cellular, and network levels. Lab Chip 15, 2767-2780.

O'Hearn, T.M., Sadda, S.R., Weiland, J.D., Maia, M., Margalit, E., Humayun, M.S., 2006. Electrical stimulation in normal and retinal degeneration (rd1) isolated mouse retina. Vision Res. 46, 3198-3204.

Peterson, B.B., Dacey, D.M., 1998. Morphology of human retinal ganglion cells with intraretinal axon collaterals. Vis. Neurosci. 15, 377-387.

Radivojevic, M., Jäckel, D., Altermatt, M., Müller, J., Viswam, V., Hierlemann, A., Bakkum, D.J., 2016. Electrical Identification and Selective Microstimulation of Neuronal Compartments Based on Features of Extracellular Action Potentials. Sci. Rep. 6, 31332.

Rattay, F., 1987. Ways to approximate current-distance relations for electrically stimulated fibers. J. Theor. Biol. 125, 339-349. 
Rattay, F., Paredes, L.P., Leao, R.N., 2012. Strength-duration relationship for intra- versus extracellular stimulation with microelectrodes. Neuroscience 214, 1-13.

Rattay, F., Wenger, C., 2010. Which elements of the mammalian central nervous system are excited by low current stimulation with microelectrodes? Neuroscience 170, 399-407.

Rhoades, C.E., Shah, N.P., Manookin, M.B., Brackbill, N., Kling, A., Goetz, G., Sher, A., Litke, A.M., Chichilnisky, E.J., 2019. Unusual Physiological Properties of Smooth Monostratified Ganglion Cell Types in Primate Retina. Neuron 103, 658-672.e6.

Royeck, M., Horstmann, M.-T., Remy, S., Reitze, M., Yaari, Y., Beck, H., 2008. Role of axonal NaV1.6 sodium channels in action potential initiation of CA1 pyramidal neurons. J. Neurophysiol. 100, 2361-2380.

Sekirnjak, C., Hottowy, P., Sher, A., Dabrowski, W., Litke, A.M., Chichilnisky, E.J., 2006. Electrical stimulation of mammalian retinal ganglion cells with multielectrode arrays. J. Neurophysiol. 95, 3311-3327.

Sekirnjak, C., Hottowy, P., Sher, A., Dabrowski, W., Litke, A.M., Chichilnisky, E.J., 2008. High-resolution electrical stimulation of primate retina for epiretinal implant design. J. Neurosci. 28, 4446-4456.

Sekirnjak, C., Hulse, C., Jepson, L.H., Hottowy, P., Sher, A., Dabrowski, W., Litke, A.M., Chichilnisky, E.J., 2009. Loss of responses to visual but not electrical stimulation in ganglion cells of rats with severe photoreceptor degeneration. J. Neurophysiol. 102, 3260-3269.

Shah, N., Madugula, S., Hottowy, P., Sher, A., Litke, A., Paninski, L., Chichilnisky, E.J., 2019. Efficient characterization of electrically evoked responses for neural interfaces. Adv. Neural Inf. Process. Syst. 32, 14444-14458.

Shah, N.P., Madugula, S., Grosberg, L., Mena, G., Tandon, P., Hottowy, P., Sher, A., Litke, A., Mitra, S., Chichilnisky, E.J., 2019. Optimization of Electrical Stimulation for a High-Fidelity Artificial Retina. In: 2019 9th International IEEE/EMBS Conference on Neural Engineering (NER). pp. 714-718.

Suzuki, S., Humayun, M.S., Weiland, J.D., Chen, S.-J., Margalit, E., Piyathaisere, D.V., de Juan, E., Jr, 2004. Comparison of electrical stimulation thresholds in normal and retinal degenerated mouse retina. Jpn. J. Ophthalmol. 48, 345-349.

Tandon, P., Bhaskhar, N., Shah, N., Madugula, S., Grosberg, L., Fan, V.H., Hottowy, P., Sher, A., Litke, A.M., Chichilnisky, E.J., Mitra, S., 2021. Automatic Identification and Avoidance of Axon Bundle Activation for Epiretinal Prosthesis. bioRxiv.

Tsai, D., Chen, S., Protti, D.A., Morley, J.W., Suaning, G.J., Lovell, N.H., 2012. Responses of retinal ganglion cells to extracellular electrical stimulation, from single cell to population: model-based analysis. PLoS One 7, e53357.

Van Wart, A., Matthews, G., 2006. Expression of sodium channels Nav1.2 and Nav1.6 during postnatal development of the retina. Neurosci. Lett. 403, 315-317.

Viswam, V., Obien, M.E.J., Franke, F., Frey, U., Hierlemann, A., 2019. Optimal Electrode Size for Multi-Scale Extracellular-Potential Recording From Neuronal Assemblies. Front. Neurosci. 13, 385.

Wang, B., Weiland, J.D., 2015. Resistivity profiles of wild-type, rd1, and rd10 mouse retina. In: 2015 37th Annual International Conference of the IEEE Engineering in Medicine and Biology Society (EMBC). pp. 1650-1653.

Warland, D.K., Reinagel, P., Meister, M., 1997. Decoding visual information from a population of retinal ganglion cells. J. Neurophysiol. 78, 2336-2350.

Watanabe, M., Rodieck, R.W., 1989. Parasol and midget ganglion cells of the primate retina. J. Comp. Neurol. 289, 434-454.

Werginz, P., Raghuram, V., Fried, S.I., 2020. The relationship between morphological properties and thresholds to extracellular electric stimulation in a RGCs. J. Neural Eng. 17, 045015. 

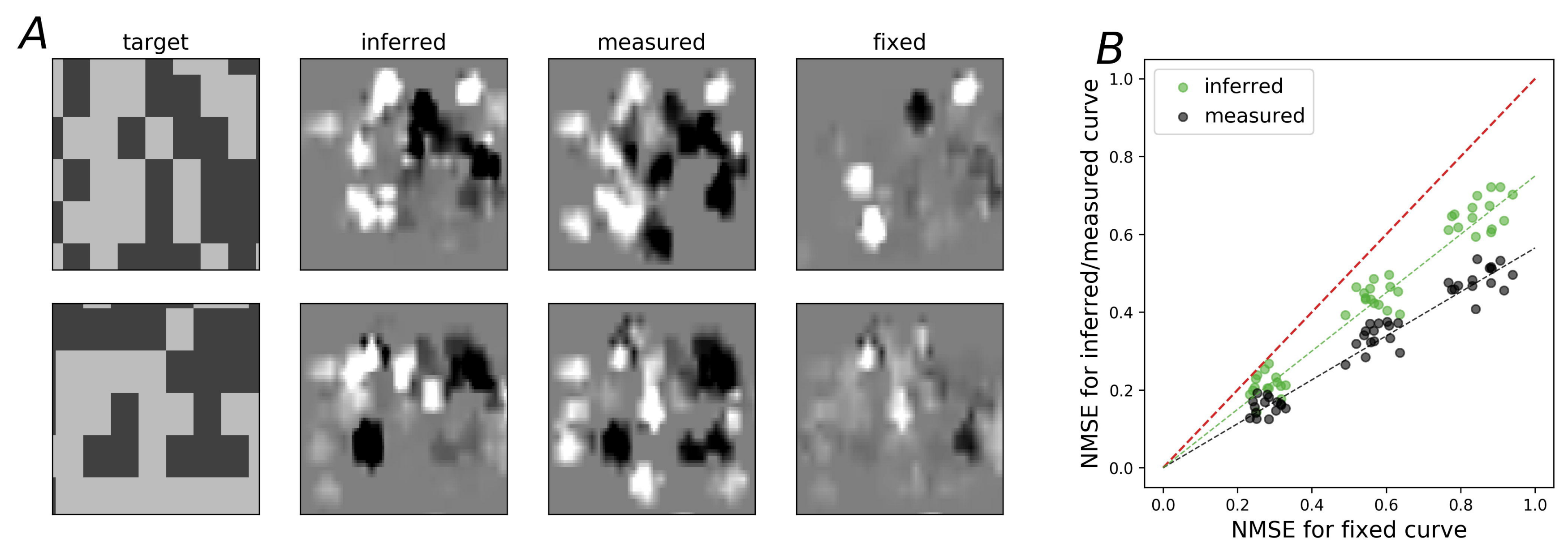
A

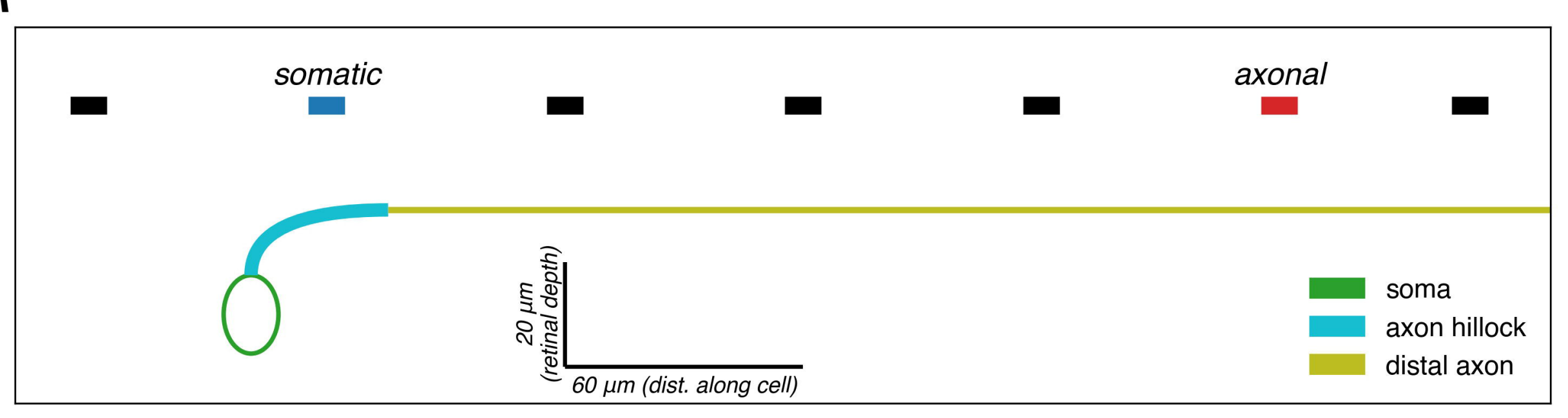

C

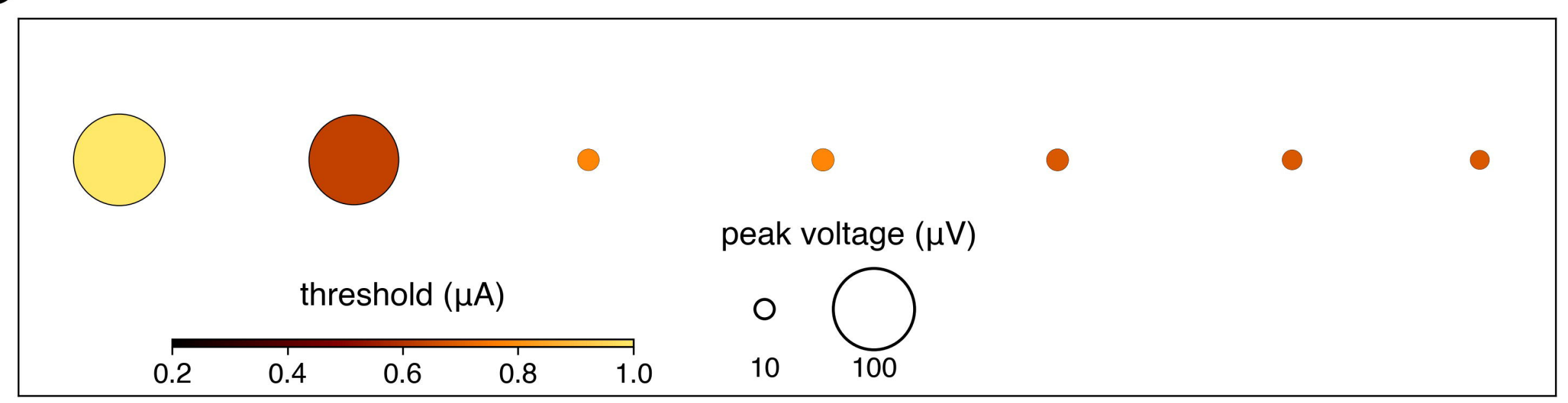

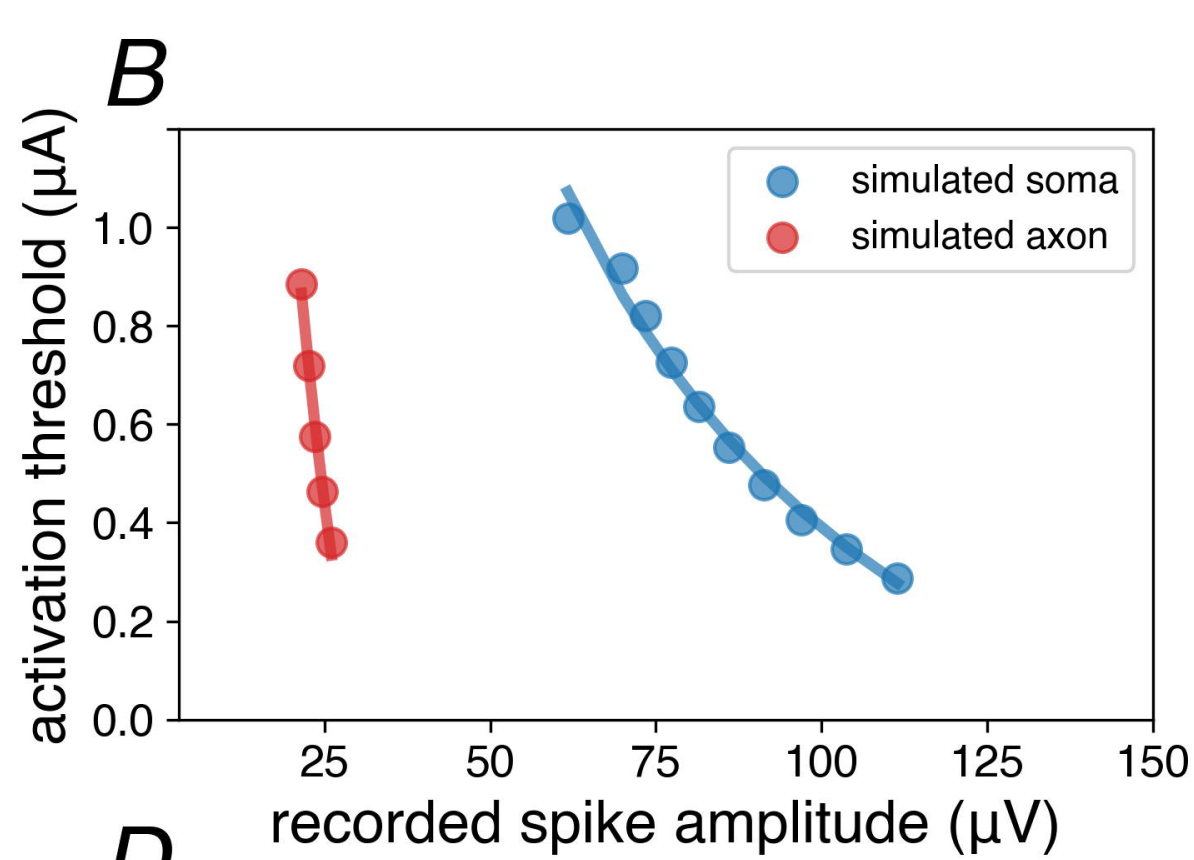

$D$ recorded spike amplitude $(\mu \mathrm{V})$

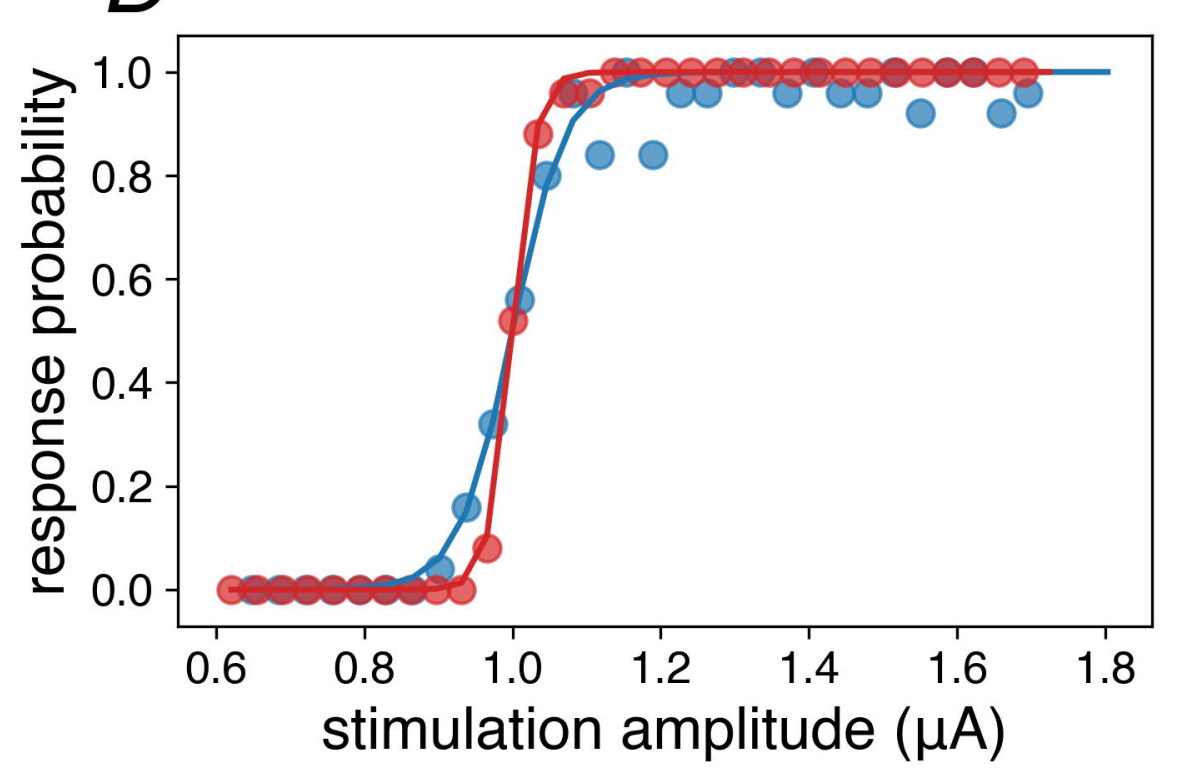



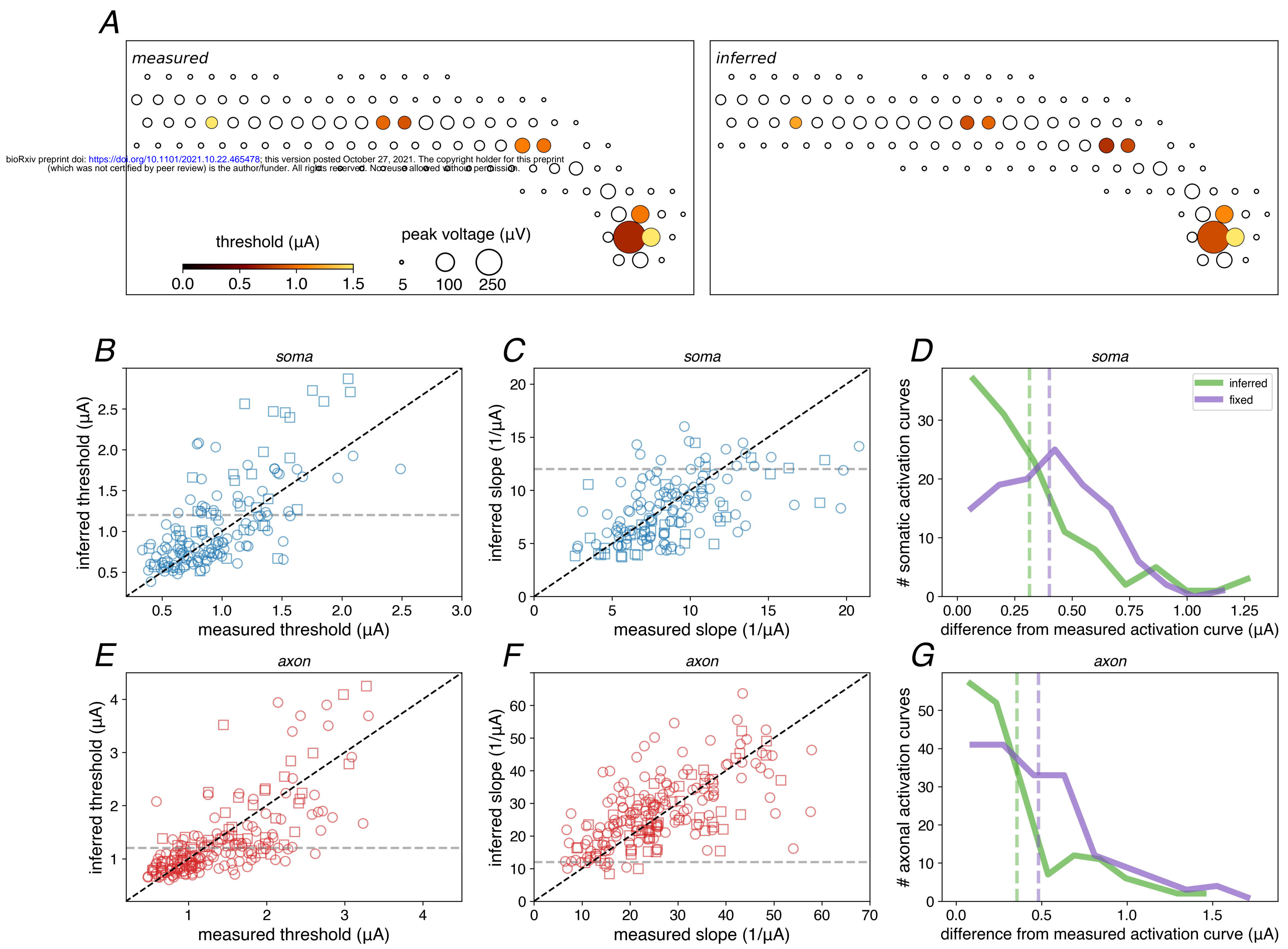
A
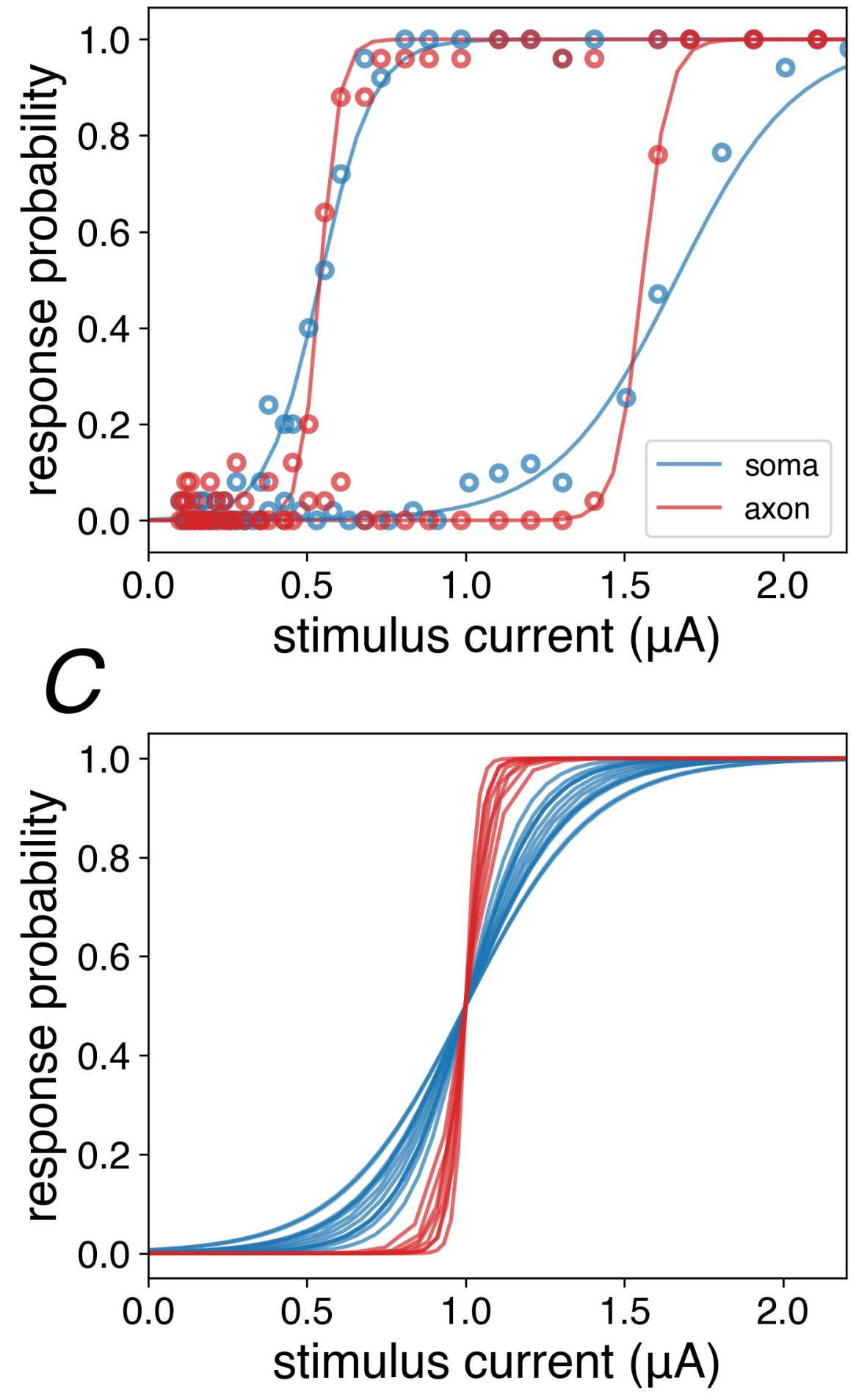

B
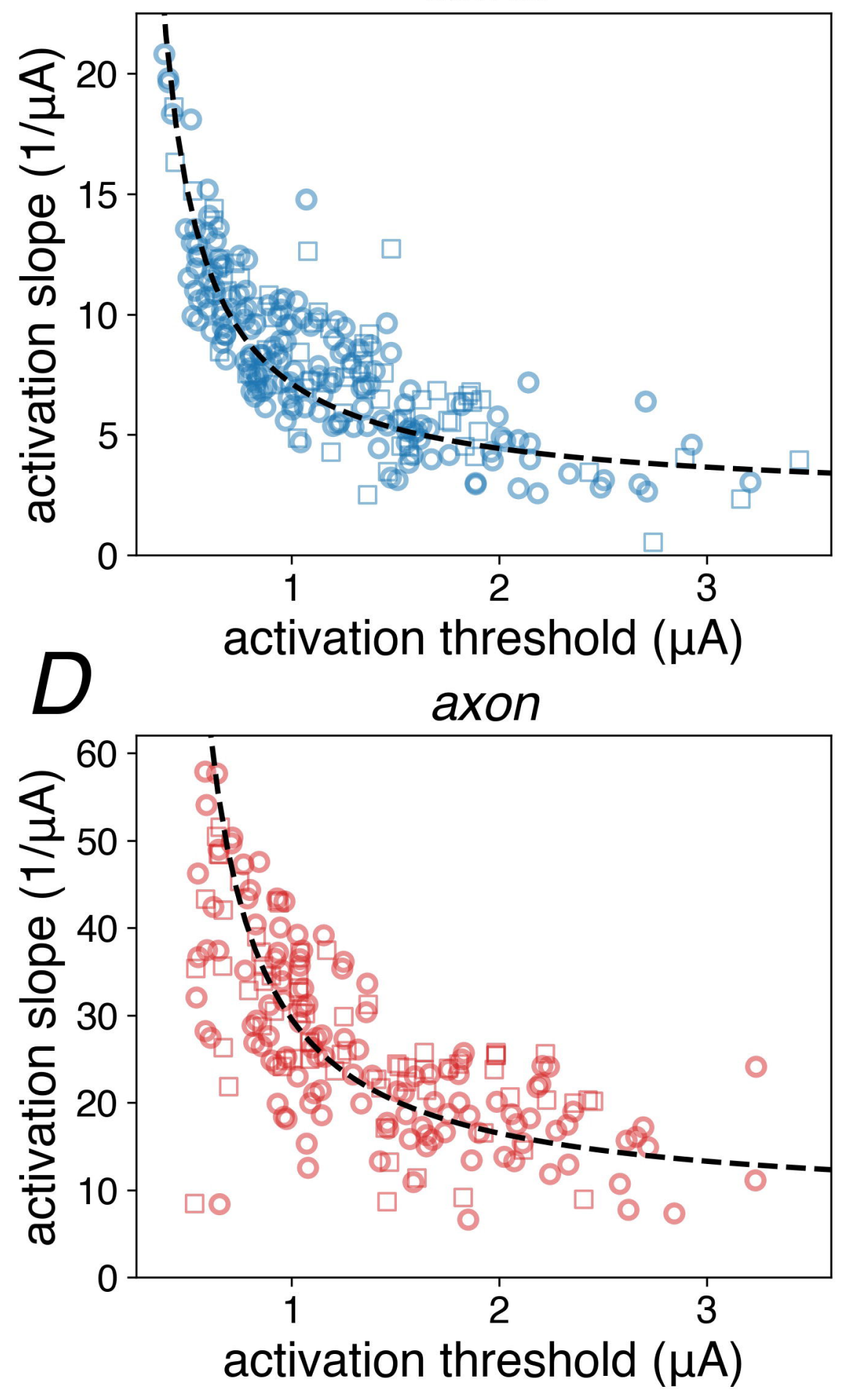

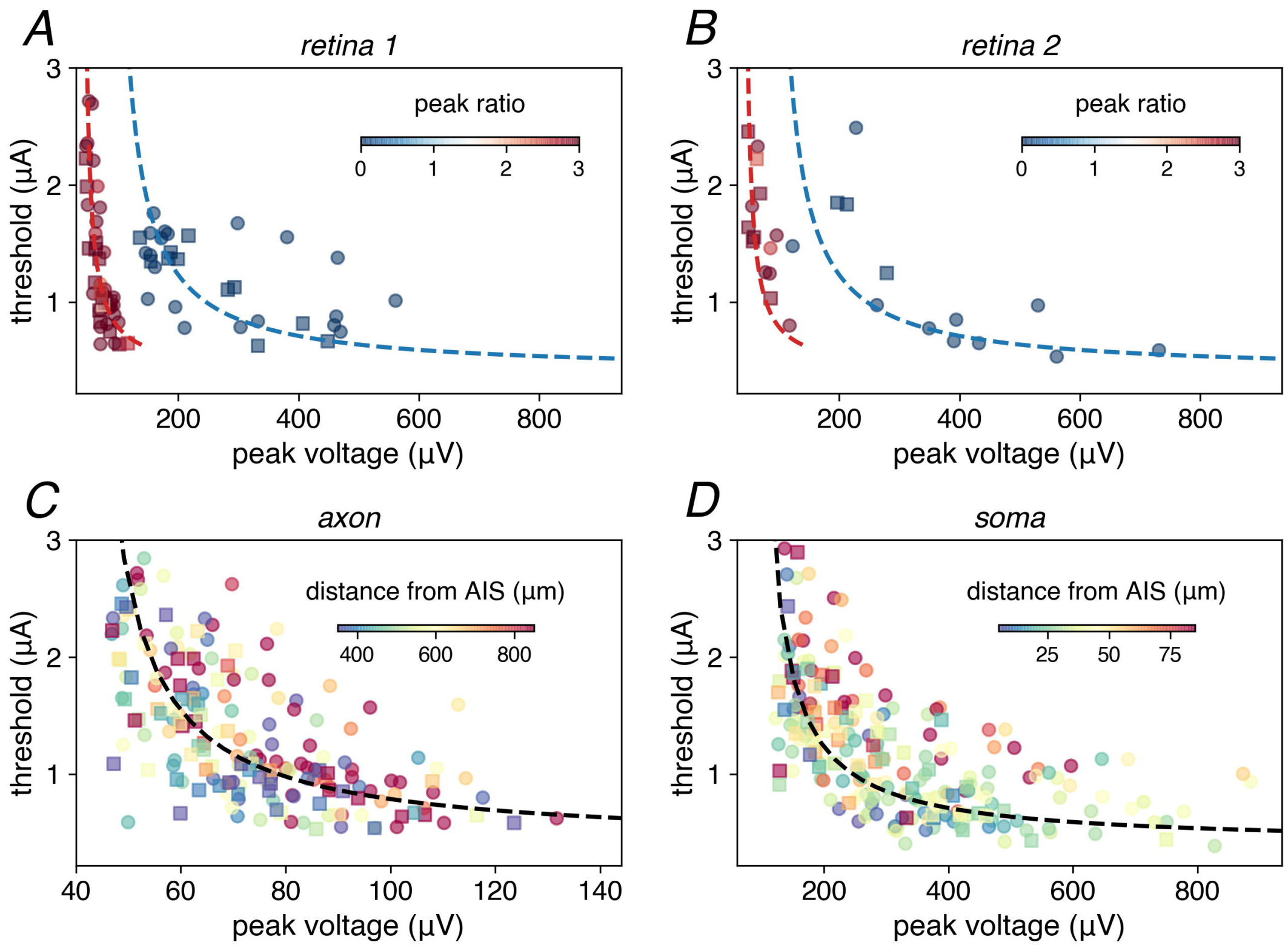


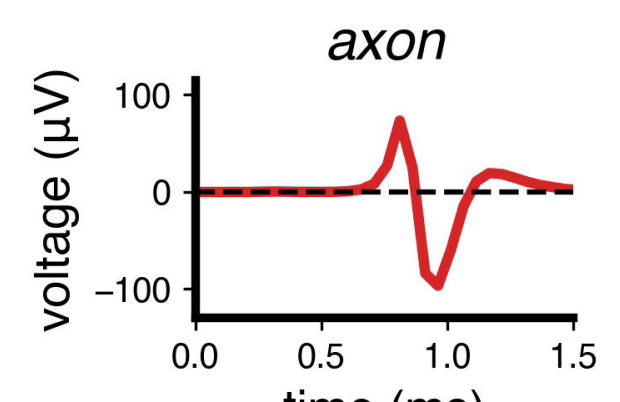

$A_{\text {ON Parasol }}^{\text {time (ms) }}$

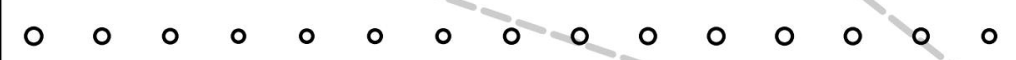

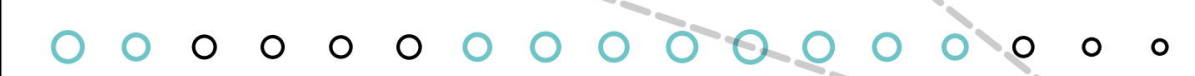

0000000000000000000

0.000000000000000000

0
0

$\underbrace{}_{0.0}$
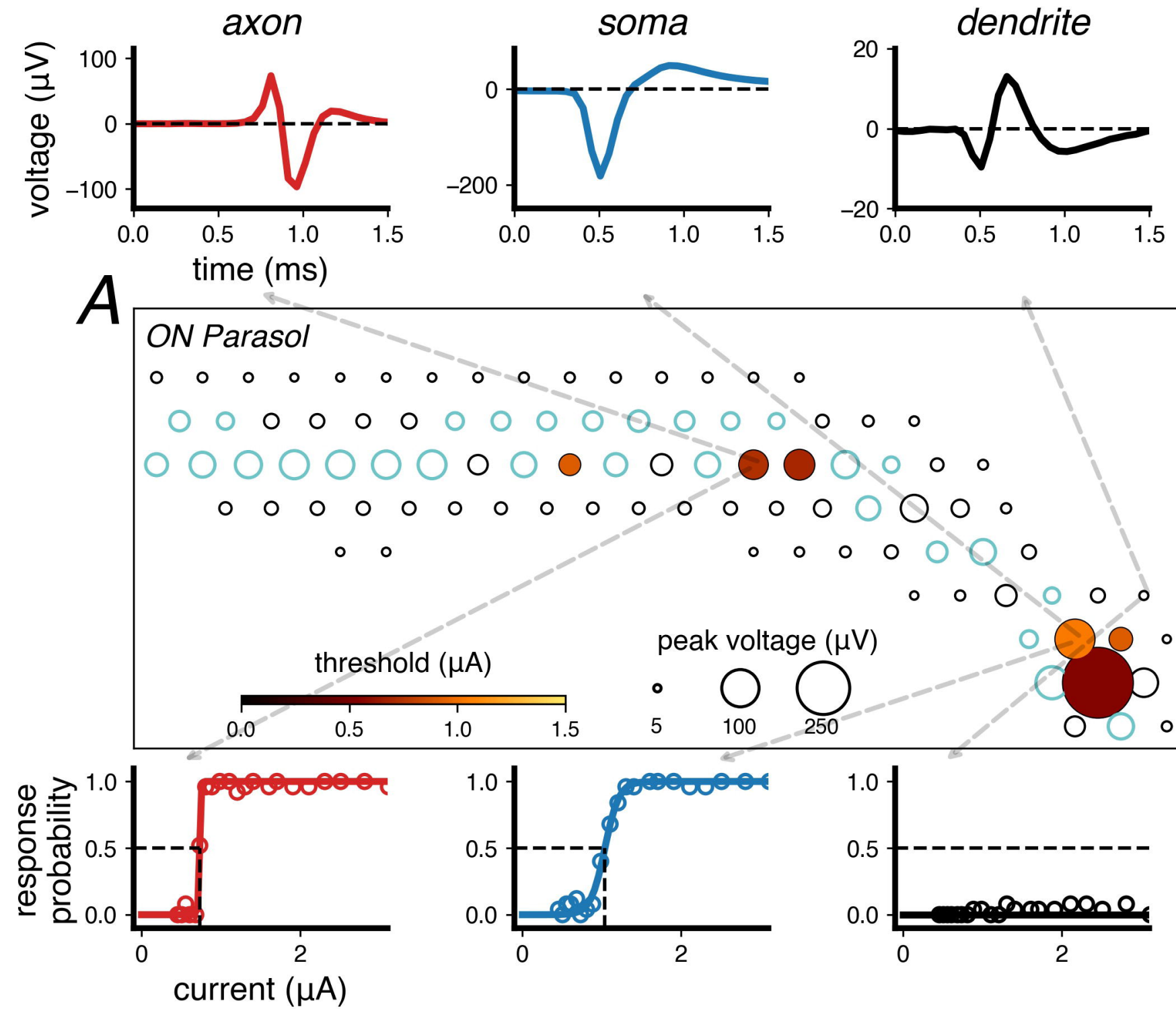

current $(\mu \mathrm{A})$

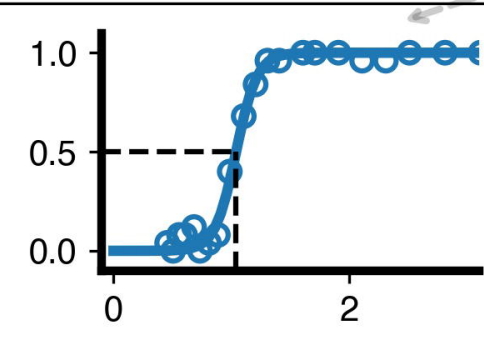

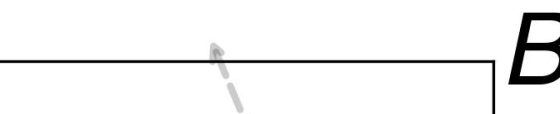

$B$

OFF Parasol

0 10

0 (1) 0

0000

- 00000

$\circ \bigcirc \bigcirc$

0

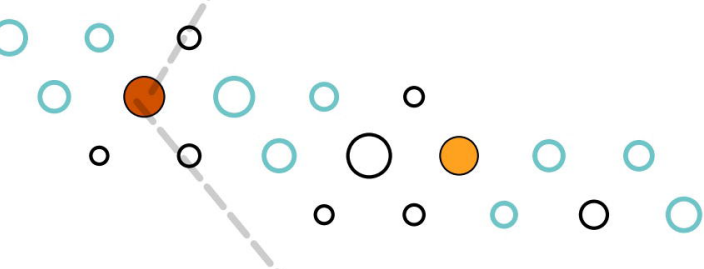
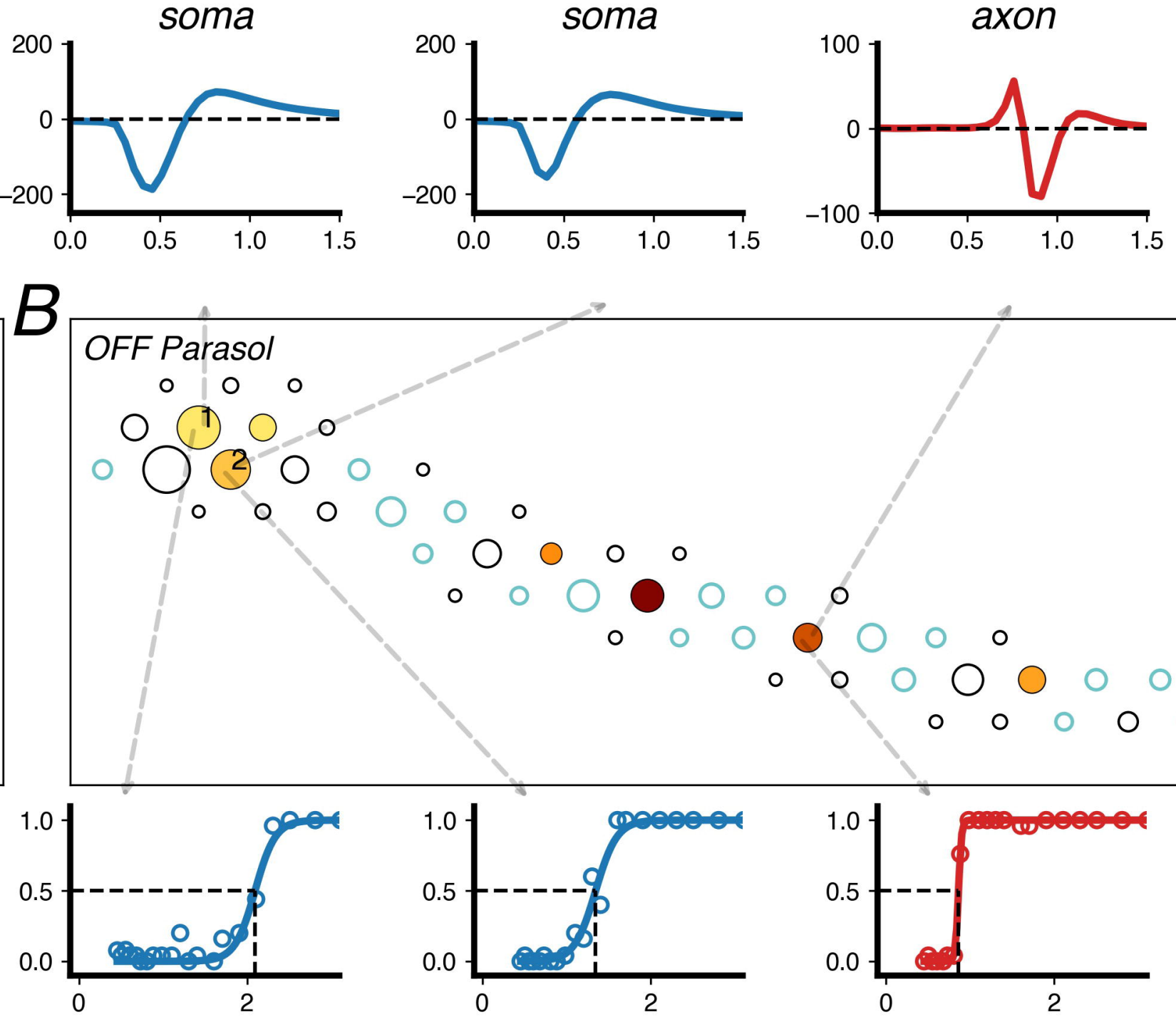